\title{
Mass Spectrometry-Based Metabolic Fingerprinting Contributes to Unveil the Role of RSUME in Renal Cell Carcinoma Cell Metabolism
}

\author{
Manuela R. Martinefski, ${ }^{\perp}$ Belén Elguero, ${ }^{\perp}$ María Elena Knott, David Gonilski, Lucas Tedesco, \\ Juan M. Gurevich Messina, Cora Pollak, Eduardo Arzt, and María Eugenia Monge*
}

Cite This: https://dx.doi.org/10.1021/acs.jproteome.0c00655

Read Online

ACCESS | Lلll Metrics \& More | 回 Article Recommendations | st Supporting Information

ABSTRACT: Clear cell renal cell carcinoma (ccRCC) is a heterogeneous disease with $50-80 \%$ patients exhibiting mutations in the von Hippel-Lindau (VHL) gene. RSUME (RWD domain (termed after three major RWD-containing proteins: RING fingercontaining proteins, WD-repeat-containing proteins, and yeast DEAD (DEXD)-like helicases)-containing protein small ubiquitinrelated modifier (SUMO) enhancer) acts as a negative regulator of VHL function in normoxia. A discovery-based metabolomics approach was developed by means of ultraperformance liquid chromatography coupled to quadrupole time-of-flight mass spectrometry (MS) for fingerprinting the endometabolome of a human ccRCC cell line 786-O and three other transformed cell

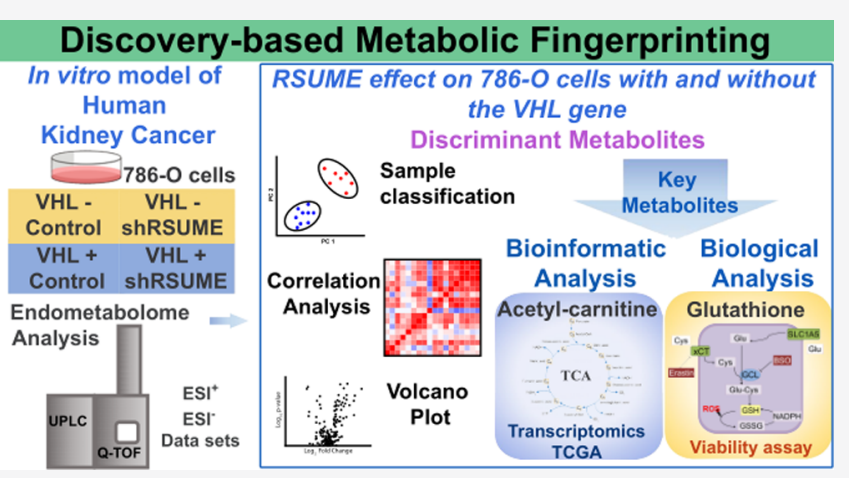
systems $(n=102)$ with different expressions of RSUME and VHL.

Cross-validated orthogonal projection to latent structures discriminant analysis models were built on positive, negative, and a combination of positive- and negative-ion mode MS data sets. Discriminant feature panels selected by an iterative multivariate classification allowed differentiating cells with different expressions of RSUME and VHL. Fifteen identified discriminant metabolites with level 1, including glutathione, butyrylcarnitine, and acetylcarnitine, contributed to understand the role of RSUME in ccRCC. Altered pathways associated with the RSUME expression were validated by biological and bioinformatics analyses. Combined results showed that in the absence of VHL, RSUME is involved in the downregulation of the antioxidant defense system, whereas in the presence of VHL, it acts in rerouting energy-related pathways, negatively modulating the lipid utilization, and positively modulating the fatty acid synthesis, which may promote deposition in droplets.

KEYWORDS: in vitro cell culture, RSUME, VHL, metabolomics, ultraperformance liquid chromatography-mass spectrometry, clear cell renal cell carcinoma, metabolic fingerprinting

\section{INTRODUCTION}

Clear cell renal cell carcinoma (ccRCC) is the most common histological subtype of RCC accounting for $75 \%$ of cases, and it is considered as a glycolytic and lipogenic tumor. ${ }^{1-4}$ ccRCC is a heterogeneous disease with $50-80 \%$ patients exhibiting mutations in the von Hippel-Lindau (VHL) gene, which is involved in the expression of hypoxia-inducible factors $1 \alpha$ and $2 \alpha$ (HIF- $1 \alpha$ and $-2 \alpha){ }^{5-7}$ HIFs are heterodimeric transcription factors that induce essential genes for the cellular and systemic homeostatic response to oxygen availability. ${ }^{8}$ When cancer cells starve for oxygen, the VHL tumor suppressor cannot target the HIFs for proteolysis, and stabilization of HIF- $1 \alpha$ and $-2 \alpha$ increases the glycolytic flux associated with tumor progression. ${ }^{9}$

RSUME (RWD domain (termed after three major RWDcontaining proteins: RING finger-containing proteins, WDrepeat-containing proteins, and yeast DEAD (DEXD)-like helicases)-containing protein small ubiquitin-related modifier (SUMO) enhancer) is the product of the RWDD3 gene. It was isolated from highly tumorigenic and angiogenic cells and is expressed in several types of tumors with high angiogenic phenotype. ${ }^{10-16}$ High RSUME expression has been associated with poor prognosis in ccRCC, and it acts as a negative regulator of VHL function in normoxia, promoting HIF- $\alpha$ stabilization $^{12,17}$ and activating HIF target genes, such as VEGF and GLUT1. ${ }^{11,12,17}$ Moreover, high RSUME expression has been correlated with high-grade ccRCC tumors. ${ }^{17}$ Considering that RSUME is implicated on HIF deregulation in ccRCC and that the HIF is a key regulator of metabolic reprogramming in this cancer type, we hypothesize that

Received: August 22, 2020 


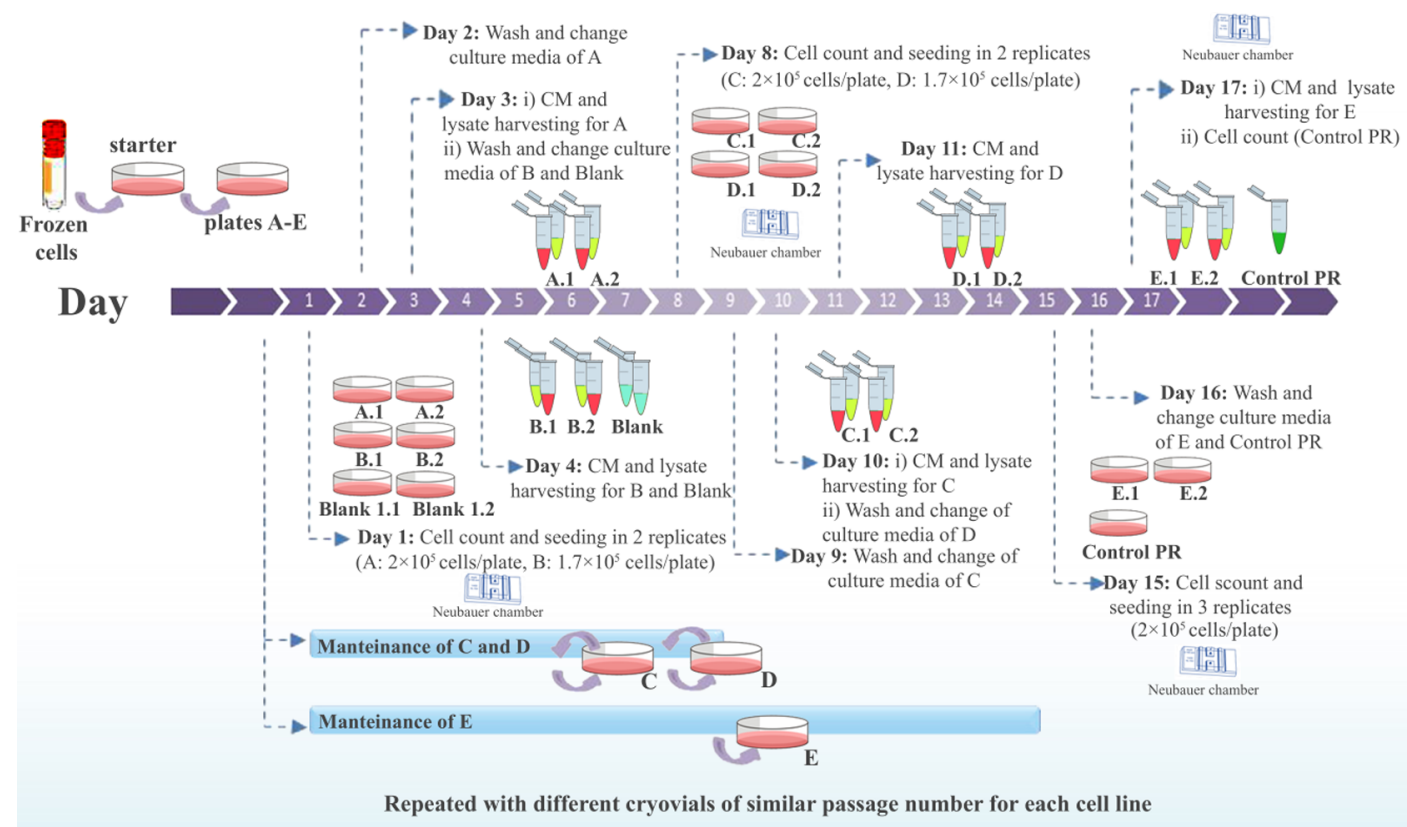

Figure 1. Scheme illustrating cell culture and sample collection for the different cell lines studied. CM: conditioned media; control PR: internal control of proliferation rate.

RSUME participates in metabolic changes in ccRCC tumor progression.

Metabolic reprogramming is an accepted hallmark associated with uncontrolled growth and spread of abnormal human cells. ${ }^{18}$ Interactions between different cancer cells with the surrounding and the molecules in the microenvironment characterize the tumor metabolome. Thus, fingerprints provided by changes of metabolite relative levels can offer a functional readout of the cellular state and biochemical activity. ${ }^{19}$ In this sense, untargeted metabolite profiling is an analytical strategy that contributes to understand the biological mechanisms and cellular pathways involved in cell biology. ${ }^{19}$

In vitro cell models are controlled systems that allow studying the role of particular genes or proteins, ${ }^{20}$ drug screening, ${ }^{21,22}$ and also the interrogation of both the intracellular (fingerprint) and extracellular (footprint) metabolome. ${ }^{23}$ In this context, ultraperformance liquid chromatography-high-resolution mass spectrometry (UPLC-HRMS) appears as a sensitive and versatile analytical platform to globally profile metabolites from in vitro models. Our research group has previously investigated the exometabolome of two human ccRCC cell lines with different VHL mutations and a nontumor human renal cell line by means of a discovery-based metabolic footprinting approach to find potential biomarkers for kidney cancer detection. ${ }^{24}$ However, the effect of RSUME on ccRCC cells with different VHL statuses using an untargeted fingerprinting approach has not been reported up to date.

In this study, we have optimized a protocol for harvesting, quenching, extracting, lyophilizing, reconstituting, and profiling the endometabolome derived from the human ccRCC cell line $786-\mathrm{O}$ deficient in VHL $\left(\mathrm{VHL}^{-/-}\right),{ }^{25}$ as well as from three other transformed cell systems $(n=102)$ with different expressions of RSUME and VHL. We used a discovery-based fingerprinting approach by means of UPLC coupled to quadrupole time-of-flight MS (UPLC-QTOF-MS) combined with cross-validated orthogonal projection to latent structures discriminant analysis (oPLS-DA) models. The metabolite panels selected by an iterative multivariate classification allowed differentiating cells with different expressions of RSUME and VHL. Discriminant metabolites identified with level $1^{26,27}$ provided clues to understand altered pathways associated with RSUME expression in 786-O and 786-O VHL cells. These pathways were validated by biological and bioinformatics analyses and indicate a disruption of the cell antioxidant defense and modulation of the fatty acid metabolism depending on the interaction of RSUME and VHL statuses.

\section{MATERIALS AND METHODS}

\section{Chemicals}

Optima LC-MS-grade acetonitrile and methanol, as well as analytical grade formic acid, were purchased from Fisher Chemical (Raleigh, NC, USA). Ultrapure water with 18.2 M . $\mathrm{cm}$ resistivity (Thermo Scientific Barnstead Micropure UV ultrapure water system, Sunnyvale, CA, USA) was used to prepare mobile phases and solutions. Leucine enkephalin was purchased from Waters Corp. (Milford, MA, USA). Dulbecco's modified Eagle's medium (DMEM) powder culture media, 1\% phosphate-buffered saline (PBS), 4-(2-hydroxyethyl)-1-piperazineethanesulfonic acid (HEPES), streptomycin, penicillin, and L-glutamine were purchased from GIBCO Thermo Fisher Scientific (Waltham, MA, USA). Fetal bovine serum (FBS) was purchased from Natocor S.A. (Córdoba, Argentina), and $\mathrm{NaHCO}_{3}$ and sodium glutamate 1-hydrate $(\geq 99 \%)$ were purchased from Biopack (Buenos Aires, Argentina). Boc-Lalanine $(\geq 99 \%)$, buthionine sulfoximine (BSO) ( $\geq 97 \%)$, butyryl-L-carnitine $(\geq 97 \%)$, erastin $(\geq 98 \%)$, isobutyryl-Lcarnitine $(\geq 97 \%)$, L- $(+)$-lactic acid $(\geq 98 \%)$, L-glutathione oxidized ( $\geq 98 \%)$, L-glutathione reduced $(\geq 98 \%)$, L-( -$)$-malic acid $(\geq 95 \%)$, L-leucine $(\geq 98 \%)$, L-phenylalanine ( $\geq 98 \%)$, lysophosphatidylcholine USP standard (PC 0:0/16:0 65\%), $O$-acetyl-L-carnitine hydrochloride $(\geq 98 \%)$, phosphocholine chloride calcium salt tetrahydrate $(\geq 97 \%)$, taurine $(\geq 99 \%)$, 
and L-tryptophan ( $\geq 98 \%)$ were purchased from Sigma-Aldrich (St. Louis, MO, USA). L-isoleucine ( $\geq 98 \%$ ) was supplied by Fluka (Steinheim, Germany). sn-glycero-3-Phosphocholine $(\geq 95 \%)$ was purchased from Cayman Chemical (Ann Arbor, MI, USA). Crystal violet was supplied by ChemCruz (Dallas, Texas, USA). N-Acetyl-phenylalanine was chemically synthesized and characterized in our laboratory. ${ }^{24}$

\section{In Vitro Model-Cell Culture Design}

The 786-O cell line, which derives from a primary ccRCC tumor and has a deletion of the gene encoding the VHL protein $\left(\mathrm{VHL}^{-/-}\right)$, was obtained from $\mathrm{R}$ Voest, University Medical Center Utrecht. Stable clones were obtained as described elsewhere, ${ }^{12}$ transfecting the RCC 786-O cells with pcDNA3-shScramble or shRSUME or pcDNA3-Flag-VHLshScramble or shRSUME, leading to four cell lines: the RCC 786-O cells silenced for endogenous RSUME (786-O shRSUME), the RCC 786-O cells with endogenous RSUME levels (786-O scramble), the RCC 786-O cells re-expressing VHL and silenced for endogenous RSUME (786-O VHL shRSUME), and the RCC 786-O cells re-expressing VHL with endogenous RSUME levels (786-O VHL scramble). Cells were cultured in DMEM ( $\mathrm{pH}=7.3$ ) supplemented with $10 \%$ FBS, $2.2 \mathrm{~g} \mathrm{~L}^{-1} \mathrm{NaHCO}_{3}, 10 \mathrm{mM}$ HEPES, $4 \mathrm{mM}$ L-glutamine, $100 \mathrm{U}$ $\mathrm{mL}^{-1}$ penicillin, and $100 \mathrm{mg} \mathrm{mL}^{-1}$ streptomycin. All cells were cultured at $37{ }^{\circ} \mathrm{C}$ in a humidified atmosphere of $5 \% \mathrm{CO}_{2}$. The cells were regularly tested for mycoplasma. Three different culture media preparation batches were used. The protocol designed for cell culture and endometabolome analysis generated 30 samples for each cell line (Figure 1). Three cryovials with a similar cell passage number were thawed for each cell line. Cells from each cryovial were split into five plates, treated independently during the experiments, and cultured under maintenance conditions until use. The cells were counted and seeded for each independent plate. After cell counting, two plates were plated for each cell line, except for plate \#5, which was split in triplicates to use one of them as an internal control of the proliferation rate (control PR) (Figure 1). Culture media blanks were obtained by incubating the cell culture media using the same protocol used for cell lines (Figure 1).

\section{Sample Collection for Endometabolome Analysis}

Cells were counted using a Neubauer chamber and cultured in $10 \mathrm{~cm}$ plates. Once $80 \%$ confluence was reached, the cells were gently washed three times for $10 \mathrm{~min}$ with the DMEM culture media, without FBS, antibiotics, and phenol red. Then, cell monolayers were incubated overnight with $5 \mathrm{~mL}$ of culture media under starving conditions ${ }^{28}$ (without FBS) and without phenol red or antibiotics. Conditioned media (culture media modified by overnight cell incubation) were harvested and aliquoted in conic microtubes of $400 \mu \mathrm{L}$ and immediately frozen at $-80{ }^{\circ} \mathrm{C}$ for further analysis. For endometabolome analysis, the cells were washed twice with $5 \mathrm{~mL}$ of PBS solution and once with $5 \mathrm{~mL}$ of ultrapure water at $37^{\circ} \mathrm{C}$ in a fast way to reduce cross-contamination and matrix effects while minimizing perturbation of the metabolic steady state of the cells. ${ }^{29}$ Subsequently, the cells were carefully scraped with $2 \mathrm{~mL}$ of cold $\left(-20{ }^{\circ} \mathrm{C}\right)$ methanol/acetonitrile $(50: 50 \mathrm{v} / \mathrm{v}) ; 1.4 \mathrm{~mL}$ of the resulted suspension was sonicated on a water-ice bath to complete cell lysis, incubated for $15 \mathrm{~min}$ at $0{ }^{\circ} \mathrm{C}$, and centrifuged at $16,000 \mathrm{~g}$ for $20 \mathrm{~min}$ at $4{ }^{\circ} \mathrm{C}$. The supernatant was split into two aliquots of $640 \mu \mathrm{L}$ and frozen at $-80{ }^{\circ} \mathrm{C}$ after the addition of $800 \mu \mathrm{L}$ of ultrapure water in each tube. One plate (control PR) was separated before scraping and used to count cells. Process blanks were prepared using plates without cells that were subjected to the sample preparation protocol described above. Lyophilization blanks consisted of ultrapure water. Samples and blanks were stored at $-80{ }^{\circ} \mathrm{C}$ until lyophilization.

\section{Sample Preparation}

Samples and blanks were lyophilized at $-80{ }^{\circ} \mathrm{C}$ and $50 \mathrm{mTorr}$ for $48 \mathrm{~h}$ using a Telstar LYOQuest- 85 freeze-dryer (Telstar, Madrid, Spain) and stored at $-80{ }^{\circ} \mathrm{C}$ until use. For each sample, one aliquot was used for endometabolome analysis and the other was stored at $-80{ }^{\circ} \mathrm{C}$ for future analyses. The samples were reconstituted the same day in $60 \mu \mathrm{L}$ of water/ methanol $(80: 20 \mathrm{v} / \mathrm{v})$ solution to reach a final concentration of $1 \times 10^{4}$ cells $\mu \mathrm{L}^{-1}$, vortex-mixed for $15 \mathrm{~s}$, and centrifuged at $15,000 \mathrm{~g}$ for $20 \mathrm{~min}$ at $4{ }^{\circ} \mathrm{C}$. Intrastudy quality control (QC) samples were prepared by pooling $5 \mu \mathrm{L}$ of aliquots of each sample, vortex-mixing for $30 \mathrm{~s}$, and subsequently splitting into six microtubes that were stored at $-80{ }^{\circ} \mathrm{C}$ together with samples until use. Quality assurance procedures were applied to verify the performance of the analytical method and lack of contamination before analysis. A system suitability QC sample (SSS) was used to verify the stability of retention times, peak shapes, and areas before sample analysis, in the middle, and at the end of each sample batch. The SSS consisted of N-acetylphenylalanine ( 5 and $25 \mu \mathrm{M}$ for positive and negative ion modes, respectively) and Boc-L-alanine (20 and $100 \mu \mathrm{M}$ for positive and negative ion modes, respectively). Out of 120 samples, 18 were used for method development and optimization. A total of 102 randomized samples (26 for each class except for the 786-O VHL scramble cell line with 24 samples) were analyzed by UPLC-QTOF-MS after conditioning the analytical platform with intrastudy QC samples.

\section{Ultraperformance Liquid \\ Chromatography-High-Resolution Mass Spectrometry}

UPLC-HRMS analyses were performed using a Waters ACQUITY UPLC I Class system fitted with a Waters ACQUITY UPLC BEH $C_{18}$ column $(2.1 \times 100 \mathrm{~mm}, 1.7 \mu \mathrm{m}$ particle size, Waters Corporation, Milford, MA, USA) coupled to a Xevo G2S QTOF mass spectrometer (Waters Corporation, Manchester, UK) with an electrospray ionization (ESI) source. The typical resolving power and mass accuracy of the Xevo G2S QTOF mass spectrometer were 32,000 full width at half-maximum and $0.3 \mathrm{ppm}$ at $m / z 554.2615$, respectively. The mobile phase consisted of water with $0.1 \%$ formic acid (mobile phase A) and methanol (mobile phase B). The flow rate was constant at $0.3 \mathrm{~mL} \mathrm{~min}^{-1}$, and the elution gradient was set as follows: $0-1.6 \mathrm{~min} 10 \% \mathrm{~B}$; $1.6-2 \mathrm{~min} 10-20 \% \mathrm{~B} ; 2-6 \mathrm{~min}$ $20-70 \%$ B; 6-7 min $70 \%$ B; 7-14 min 70-90\% B; $14-17.5$ $\min 90 \% \mathrm{~B} ; 17.5-18 \min 90-95 \% \mathrm{~B}$; and $18-21 \min 95 \% \mathrm{~B}$. After each sample injection, the gradient was returned to its initial conditions in $9 \mathrm{~min}$; thus, the total run time was $30 \mathrm{~min}$. The column and autosampler tray temperatures were set at 35 and $5{ }^{\circ} \mathrm{C}$, respectively. The injection volume was $2 \mu \mathrm{L}$. Data were collected in positive and negative ionization modes in separate runs and using the same chromatographic method.

The mass spectrometer was operated in positive and negative ion modes with probe capillary voltages of 2.5 and $2.3 \mathrm{kV}$, respectively, and a sampling cone voltage of $30 \mathrm{~V}$ for both modes. The source and desolvation gas temperatures were set to 120 and $300{ }^{\circ} \mathrm{C}$, respectively. The nitrogen gas desolvation flow rate was $600 \mathrm{~L} \mathrm{~h}^{-1}$, and the cone desolvation 
flow rate was $10 \mathrm{~L} \mathrm{~h}^{-1}$. The mass spectrometer was daily calibrated across the range of $\mathrm{m} / z 50-1200$ using a $0.5 \mathrm{mM}$ sodium formate solution prepared in 2-propanol/water (90:10 $\mathrm{v} / \mathrm{v})$. Data were drift-corrected during acquisition using a leucine enkephalin reference spray infused every $45 \mathrm{~s}$ at $5 \mu \mathrm{L}$ $\min ^{-1}$ for negative ion mode $(m / z 554.2615)$ and at $2 \mu \mathrm{L}$ $\min ^{-1}$ for positive ion mode $(m / z 556.2771)$. Data were acquired in the $\mathrm{MS}^{\mathrm{E}}$ continuum mode ${ }^{30}$ in the range of $\mathrm{m} / \mathrm{z}$ $50-1200$, and the scan time was set to $0.5 \mathrm{~s}$. For UPLC-MS/ MS experiments, the product ion mass spectra were acquired with collision cell voltages between 6 and $40 \mathrm{~V}$ depending on the analyte. Ultra-high-purity argon $(\geq 99.999 \%)$ was used as the collision gas. Data acquisition and processing were carried out using MassLynx version 4.1 (Waters Corp., Milford, MA, USA). The MS data have been deposited in the MetaboLights public repository, with the data set identifier MTBLS1935 (https://www.ebi.ac.uk/metabolights/MTBLS1935).

\section{UPLC-HRMS Analysis}

Samples were randomly analyzed by UPLC-QTOF-MS within a template of intrastudy QC samples. The sample lists for UPLC-HRMS analysis were set up as follows for each analytical batch (number of injections $\times$ sample type): $2 \times$ solvent blank [water/methanol $(80: 20 \mathrm{v} / \mathrm{v})]$; $1 \times$ lyophilization blank; $1 \times$ SSS; $1 \times$ process blank; $2 \times$ intrastudy QC; $20 \times$ randomized samples with $1 \times$ intrastudy $Q C$ every five samples and $1 \times$ SSS every 10 samples; $2 \times$ intrastudy QC; $1 \times$ SSS; and $2 \times$ solvent blank [water/methanol $(80: 20 \mathrm{v} / \mathrm{v})]$. The samples were analyzed for five consecutive days for each ionization mode. The first solvent blank injection of each batch was not considered for data analysis.

\section{Data Preprocessing}

Spectral features (retention time $\left(R_{\mathrm{t}}\right), m / z$ pairs) were extracted from UPLC-QTOF-MS data using Progenesis QI version 2.1 (Nonlinear Dynamics, Waters Corp., Milford, MA, USA) with default parameters for import filter and automatic sensitivity for peak picking. The procedure included retention time alignment, peak picking, deisotoping, integration, and grouping together the adducts derived from the same compound. Based on previous findings, ${ }^{24}$ different adduct ions with $\mathrm{NaCl}$ were also considered for this step $([\mathrm{M}+x \mathrm{NaCl}$ $-\mathrm{H}]^{-},[\mathrm{M}+x \mathrm{NaCl}+\mathrm{Cl}]^{-},[\mathrm{M}+x \mathrm{NaCl}+\mathrm{H}]^{+}$, and $[\mathrm{M}+$ $\left.x \mathrm{NaCl}+\mathrm{Na}]^{+}\right)$in addition to ionic species that are typically observed in ESI.

Two feature matrices were obtained from positive and negative data sets. Different curation steps were applied to these data. First, a $100 \%$ detection rate filter was applied to intrastudy QC samples, that is, features that were not detected in all intrastudy QC samples were discarded. In addition, signals from salt clusters based on their mass defect were removed. ${ }^{31}$ As endogenous metabolites may contain phosphate, sulfur, and iodine, exhibiting high mass defect, an "inclusion list" ${ }^{31}$ was used to account for them. Subsequently, QC sample-based robust LOESS (locally estimated scatter plot smoothing) signal correction (QC-RLSC) was applied to correct for instrumental drift during analysis using intrastudy QC samples. ${ }^{32}$ Afterward, if a feature had a peak area in a sample that was 3-fold or less than the mean peak area in blanks, then its peak area was set to $0 .{ }^{33}$ Otherwise, the mean peak area in blanks was subtracted from the feature peak areas in the samples. After blank subtraction, only those features that were present in at least $80 \%$ of one group class were retained. In addition, features with RSD $>30 \%$ in intrastudy QC samples were removed. The chromatographic peak shape and the signal intensity of each feature were further evaluated for data curation. Features with signal intensity $<10^{3}$ in the continuum mass spectra or with a mass difference larger than $10 \mathrm{mDa}$ along the sample list were discarded. Finally, each feature matrix obtained after this procedure was normalized to the total peak area for each sample.

\section{Data Processing, Classification, and Prediction}

Normalized matrices (Data Set S1 in the Supporting Information) were utilized to build unsupervised and supervised multivariate statistical analysis models using MATLAB R2015a (the MathWorks, Natick, MA, USA) with the PLS Toolbox version 8.1 (Eigenvector Research, Inc., Manson, WA, USA). Principal component analysis (PCA) was used to track data quality and identify and remove outliers in the data set. Surprisingly, samples cultured with one of the three independent culture media batches were identified as outliers by PCA (Figures S1-S3), affecting 16 samples from the 786-O shRSUME cell line and 16 from the 786-O scramble cell line. Therefore, these samples were not used for further statistical analysis. oPLS-DA ${ }^{34,35}$ coupled with a genetic algorithm (GA) or an interval PLS-DA (iPLS-DA) variable selection method was applied to find feature panels that maximized the classification accuracy for these two binary comparisons: 786-O shRSUME versus $786-\mathrm{O}$ scramble and 786-O VHL shRSUME versus 786-O VHL scramble. Feature selection was applied on three matrices corresponding to positive ion mode data, negative ion mode data, and the combined data from both ion modes, leading to six different discriminant feature panels. The parameters for GA and iPLSDA variable selection are detailed in Table S1. The oPLS-DA model was cross-validated using venetian blinds with 10 data splits. Data were autoscaled prior to generating PCA and oPLS-DA models. PCA was also performed to inspect data before and after variable selection (i.e., on the curated spectral feature matrices and on the discriminant feature panels). For the different binary comparisons, the panels of discriminant features that had the lowest root-mean-square error of crossvalidation at the conclusion of the GA variable selection process were selected. However, some features were excluded for statistical analysis (see Data Analysis and Metabolite Identification Procedure section).

\section{Data Analysis and Metabolite Identification Procedure}

Fold changes were calculated as the ratio of median peak areas for the same binary comparisons: 786-O shRSUME versus 786$\mathrm{O}$ scramble and 786-O VHL shRSUME versus 786-O VHL scramble. The Shapiro-Wilk test was used to determine the normality of data distribution. Mann-Whitney $U$ tests were used to calculate the statistical significance, and $p$-values were corrected for identified metabolites using the BenjaminiHochberg ${ }^{36}$ procedure for multiple comparisons with a false discovery rate (FDR) of 0.1 .

Metabolite identification was attempted for the 75 discriminant features resulting from the GA and iPLS-DA variable selection processes. Elemental formulae were generated based on accurate masses and isotopic patterns, and mass spectral adduct ions were searched against the Human Metabolome Database (HMDB), ${ }^{37}$ Metlin, $^{38}$ and LIPID MAPS ${ }^{39}$ databases. Metabolites were identified based on accurate mass, isotopic profiles, fragmentation patterns compared to those obtained from authentic chemical standards in tandem MS experiments, and retention time matching with 


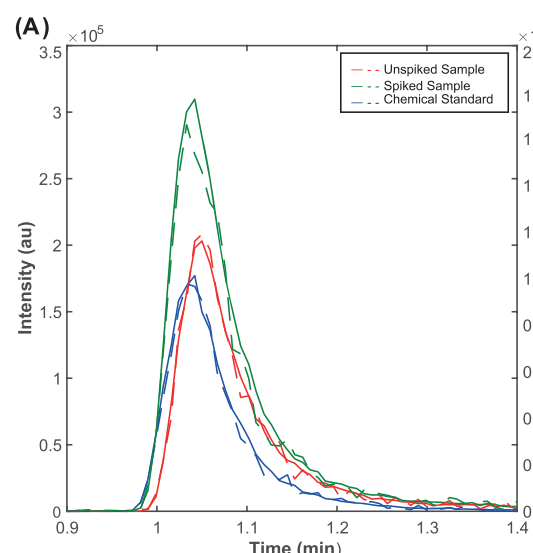

(D)

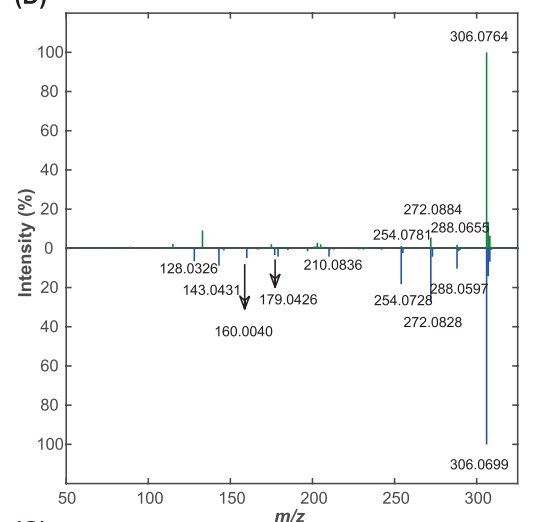

(G)

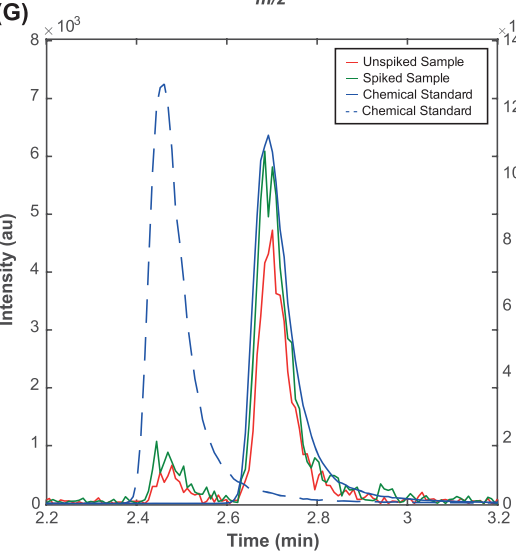

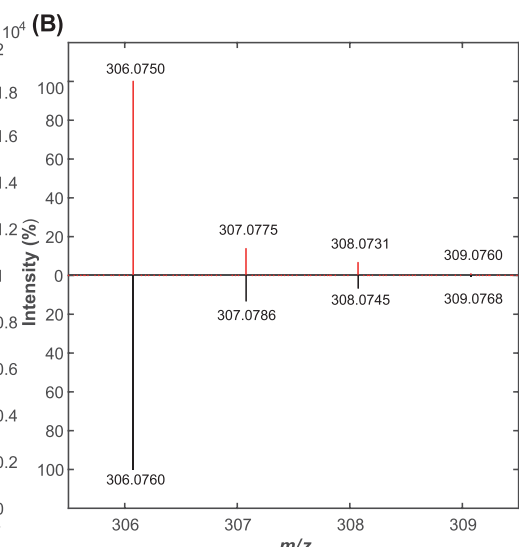

(E)
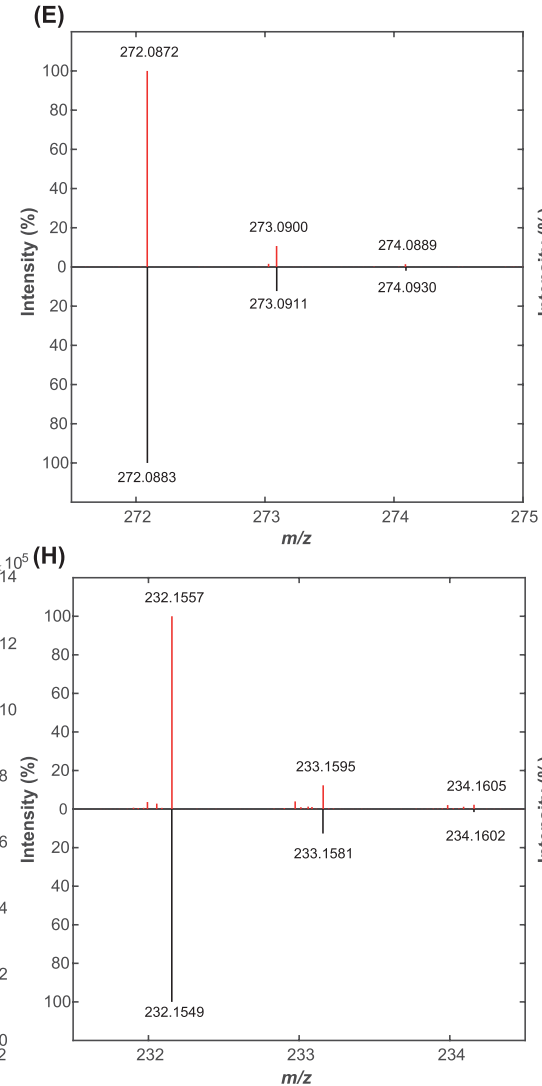

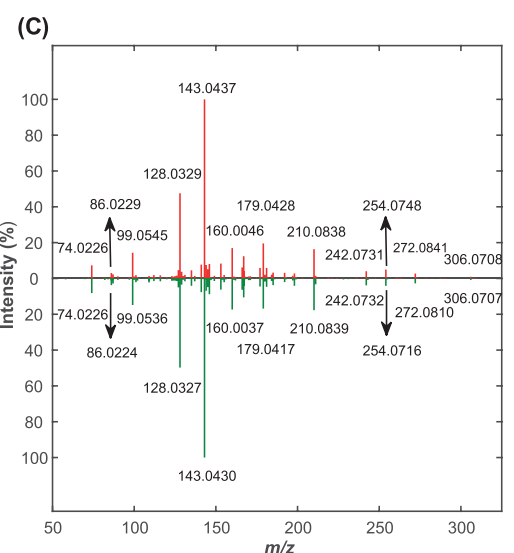

(F)
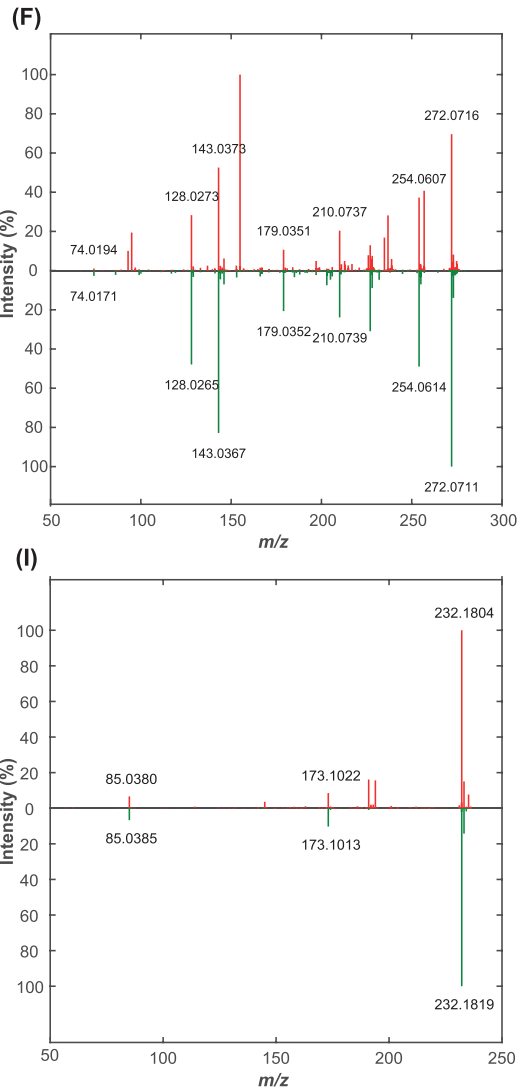

Figure 2. Discriminant metabolite identification: (A) extracted ion chromatograms for [glutathione $-\mathrm{H}]^{-}$ions at $m / z 306.0763 \pm 0.0500$ (continuous line, left $y$-axis) and for [glutathione $\left.-\mathrm{SH}_{2}-\mathrm{H}\right]^{-}$ions at $\mathrm{m} / z 272.0885 \pm 0.0500$ (dashed line, right $y$-axis) generated from nonspiked (red) and $18.9 \mu \mathrm{M}$ spiked (green) intrastudy QC samples and a $18.9 \mu \mathrm{M}$ glutathione (GSH) chemical standard solution (blue). (B) Mass spectrum for [GSH $-\mathrm{H}]^{-}$ions at $\mathrm{m} / z 306.0763$ in an intrastudy QC sample (red) and its simulated isotopic pattern (black). (C) Product ion mass spectra of $[\mathrm{GSH}-\mathrm{H}]^{-}$precursor ions for an intrastudy QC sample (red) and for a $18.9 \mu \mathrm{M}$ GSH chemical standard (green) using a collision cell voltage of $20 \mathrm{~V}$. (D) Mass spectrum for [GSH $-\mathrm{H}]^{-}$ions at $\mathrm{m} / z 306.0763$ in a $18.9 \mu \mathrm{M} \mathrm{GSH}$ chemical standard solution (green) and product ion mass spectra of $[\mathrm{GSH}-\mathrm{H}]^{-}$precursor ions for a $18.9 \mu \mathrm{M}$ GSH chemical standard solution (blue) using a collision cell voltage of $10 \mathrm{~V}$. (E) Mass spectrum for $\left[\mathrm{GSH}-\mathrm{SH}_{2}-\mathrm{H}\right]^{-}$ions at $m / z 272.0885$ in an intrastudy QC sample (red) and its simulated isotopic pattern (black). (F) Product ion mass spectra of [ $\left.\mathrm{GSH}-\mathrm{SH}_{2}-\mathrm{H}\right]^{-}$precursor ions for an intrastudy QC sample (red) and for a $18.9 \mu \mathrm{M}$ GSH chemical standard (green) using a collision cell voltage of $10 \mathrm{~V}$. (G) Extracted ion chromatograms for [butyrylcarnitine $+\mathrm{H}]^{+}$ions at $\mathrm{m} / z 232.1549 \pm 0.0500$ generated from nonspiked (red, left $y$-axis) and $10 \mu \mathrm{M}$ spiked (green, left $y$-axis) intrastudy QC samples; a $10 \mu \mathrm{M}$ butyrylcarnitine standard solution (blue solid, right $y$-axis) and a $10 \mu \mathrm{M}$ isobutyrylcarnitine standard solution (blue dash, right $y$-axis). (H) Mass spectrum for [butyrylcarnitine $+\mathrm{H}]^{+}$ions at $m / z$ 232.1535 in an intrastudy QC sample (red) and its simulated isotopic pattern (black). (I) Product ion mass spectra of [butyrylcarnitine $+\mathrm{H}]^{+}$ precursor ions for an intrastudy QC sample (red) and for a $10 \mu \mathrm{M}$ butyrylcarnitine chemical standard (green) using a collision cell voltage of $10 \mathrm{~V}$.

authentic chemical standards. Experimental MS/MS spectra were compared to databases, and for those cases in which spectra were not available, fragmentation patterns were manually interpreted for metabolite annotation. Chemical standards were prepared in ultrapure water or methanol (1-38 $\mu \mathrm{M})$ and were analyzed under identical conditions as samples for validating metabolite identification. Spiking experiments on the study samples were also conducted with chemical standards to address retention time differences caused by matrix effects ${ }^{24}$ (Figures S4 and S5). Identification of metabolites was reported according to the metabolomics standard initiative (MSI) identification standards. ${ }^{26,27}$ 
Spearman's correlation analysis and retention time difference were used to identify those features corresponding to the same compound that were not grouped by Progenesis QI, considering in-source fragmentation and additional adduct ions formed in the source. Peak areas from features that exhibited a pairwise correlation larger than 0.7 , except for two adduct ions corresponding to glutathione that were subsequently verified with chemical standards, and features with a retention time difference under $2 \mathrm{~s}$ were added and grouped under the label of "total" metabolite. Through this approach, 14 features detected in negative ion mode were identified for glutathione $(\mathrm{M})$, that is, $\left[\mathrm{M}-\mathrm{SH}_{2}-\mathrm{H}\right]^{-},[\mathrm{M}+\mathrm{Na}-2 \mathrm{H}]-$, $\left[\mathrm{M}+\mathrm{CHO}_{2} \mathrm{Na}+\mathrm{Na}-2 \mathrm{H}\right]^{-},\left[\mathrm{M}+\mathrm{H}_{3} \mathrm{PO}_{4}+\mathrm{Na}-2 \mathrm{H}\right]^{-},[\mathrm{M}$ $\left.+\mathrm{H}_{3} \mathrm{PO}_{4}-\mathrm{H}\right]-,\left[\mathrm{M}+\mathrm{NaH}_{2} \mathrm{PO}_{4}+\mathrm{Na}-2 \mathrm{H}\right]^{-},[\mathrm{M}+$ $\left.\mathrm{NaH}_{2} \mathrm{PO}_{4}+\mathrm{NaCl}+\mathrm{Na}-2 \mathrm{H}\right]^{-},\left[\mathrm{M}+2 \mathrm{NaH}_{2} \mathrm{PO}_{4}+\mathrm{Na}-\right.$ $2 \mathrm{H}]^{-},[2 \mathrm{M}+\mathrm{Na}-2 \mathrm{H}]^{-},\left[2 \mathrm{M}+\mathrm{H}_{3} \mathrm{PO}_{4}+\mathrm{Na}-2 \mathrm{H}\right]^{-},[2 \mathrm{M}+$ $\left.\mathrm{H}_{3} \mathrm{PO}_{4}-\mathrm{H}\right]^{-},[2 \mathrm{M}+3 \mathrm{Na}-4 \mathrm{H}]^{-}$, and $\left[2 \mathrm{M}+\mathrm{H}_{3} \mathrm{PO}_{4}+2 \mathrm{Na}\right.$ $-3 \mathrm{H}]^{-}$(Figures 2A-F, S4 and S15). Similarly, six features were identified for glutathione in the positive ion mode, that is, $[\mathrm{M}+\mathrm{H}]^{+},[\mathrm{M}-2 \mathrm{H}+3 \mathrm{Na}]^{+},\left[\mathrm{M}+\mathrm{NaH}_{2} \mathrm{PO}_{4}+\mathrm{Na}\right]^{+},[\mathrm{M}-$ $\left.\mathrm{C}_{5} \mathrm{H}_{7} \mathrm{NO}_{3}+\mathrm{H}\right]^{+},\left[\mathrm{M}-\mathrm{H}_{2} \mathrm{O}+\mathrm{H}\right]^{+}$, and $\left[\mathrm{M}-\mathrm{C}_{2} \mathrm{H}_{5} \mathrm{NO}_{2}+\right.$ $\mathrm{H}]^{+}$(Figures S16-S21). Two features were also identified for leucine in the positive ion mode, that is, $[\mathrm{M}+\mathrm{H}]^{+}$and $[\mathrm{M}-$ $\left.\mathrm{CH}_{2} \mathrm{O}_{2}+\mathrm{H}\right]^{+}$(Figure S22). Furthermore, one of the features was identified as HEPES, which is a nonendogenous metabolite, and was therefore discarded for analysis.

Cell Viability under Oxidative Stress and Glutathione Depletion

Two thousand cells per well were plated in triplicate for each condition in a 96-well plate in a full growth medium. After $4 \mathrm{~h}$, cells were washed with PBS before adding the full medium or the medium containing $\mathrm{H}_{2} \mathrm{O}_{2}$ at $0.1,0.5$, or $1.0 \mu \mathrm{M}$ concentrations with either $\mathrm{BSO} 1 \mathrm{mM}$ or erastin $10 \mu \mathrm{M}$. After incubation, the cells were fixed with $100 \%$ methanol, stained with a $0.01 \%$ crystal violet solution, washed, and dried. For quantification, the dye was extracted with $100 \%$ methanol and optical density was measured at $550 \mathrm{~nm}$. For $\mathrm{H}_{2} \mathrm{O}_{2}$ treatment, data were analyzed with multiple regression analysis, one-way ANOVA, and Tukey's multiple comparisons. For $\mathrm{BSO}$, erastin, BSO plus $\mathrm{H}_{2} \mathrm{O}_{2}$, and erastin plus $\mathrm{H}_{2} \mathrm{O}_{2}$ treatments, data were analyzed with an unpaired $t$-test.

\section{Bioinformatics Analysis}

The biospecimen data of somatic variants identified from exome sequencing studies of ccRCC tumors correspond to the TCGA Kidney Renal Clear Cell Carcinoma (Project ID: TCGA-KIRC) of the TCGA Resource Network. ${ }^{40}$ The data sets analyzed in this study are available at the National Cancer Institute (NIH) GDC Data Portal repository [https://portal. gdc.cancer.gov/projects/TCGA-KIRC].

A Kaplan-Meier analysis for 5 year survival conducted by The Human Protein Atlas (https://www.proteinatlas.org/ humanproteome/pathology) from 528 ccRCC patients (KIRC data set) summarizes the results from correlation analysis between the mRNA expression level of RSUME and enzymes involved in the tricarboxylic acid (TCA) cycle, fatty acid $\beta$-oxidation (FAO), or fatty acid synthesis (FAS) and patient survival. This allows dividing patients based on the mRNA expression of each gene into "low" or "high". In addition, RSUME "low" (below a cutoff value of 2.92) and RSUME "high" (over a cutoff value of 2.92) expression groups were used for further analysis in VHL wild-type patients to compare the TCA, FAO, and FAS pathway expression. The
VHL mutational analysis from tumors with sequencing data was available and downloaded from Table S1 in the study from Ricketts et al. ${ }^{41}$ To maintain data consistency, only those 463 patient samples and genes that were present in both The Human Protein Atlas and the cited Table $S 1^{41}$ were selected from the TCGA-KIRC. Only patients without mutations in the VHL gene were selected for further analysis. Gene expression of the ccRCC tumors was obtained and downloaded by using $\mathrm{R}$ Version 4.0.0 (including additional packages Bioconductor version: Release (3.11)). Gene expression was verified by using The Human Protein Atlas (https://www.proteinatlas.org/ humanproteome/pathology). Expression data is reported as the median FPKM (number Fragments Per Kilobase of exon per Million reads). Sample groups were filtered by removing those samples considered as gene expression outliers. The interquartile range (IQR) was used to find outliers defined as expression values that fall below the first quartile $(-1.5 \mathrm{IQR})$ or above the third quartile (+1.5 IQR). Data analysis from the TCGA data set was performed using R Version 4.0.0. MannWhitney $U$ tests were used to calculate the statistical significance for pairwise comparisons of gene expression values. All statistical tests were two-sided. The statistical significance level was defined as $5 \%$.

\section{RESULTS AND DISCUSSION}

\section{Metabolic Fingerprints and Sample Classification}

A protocol was developed for harvesting and extracting intracellular metabolites from an in vitro model of human renal cell lines (Figure 1), and endometabolome profiles were obtained with a reverse-phase UPLC-QTOF-MS-based analytical method. A total of 896 and 2842 features $\left(R_{t}, \mathrm{~m} / z\right.$ pairs $)$ were extracted with the Progenesis QI software from the negative and positive ion mode data, respectively. After a stringent data curation process (see the Data Preprocessing section), the numbers of features were reduced for each ion mode data set to 56 and 128, respectively (Table S2). These matrices were normalized (Data Set S1 in the Supporting Information) and used to build PCA models. The 2D score plot illustrated in Figure S1 shows that the intrastudy QC samples clustered tightly and that the intrastudy QC-variance was much smaller than the sample variance, suggesting a good reproducibility in the sample preparation method, and adequate performance of the analytical platform. In addition, the figure shows that the endometabolome can be affected by the media composition, even when using the same culture media. This result was further confirmed by analyzing the samples from cell lines 786-O shRSUME and 786-O scramble that were cultured with batches \#2 and \#3 (Figure S2). The PCA score plots clearly show that sample clustering was achieved based on the culture media batch. Conversely, sample separation was not evidenced based on the culture media batch when the same analysis was made with cell lines 786-O VHL shRSUME and 786-O VHL scramble that were cultured with batches \#1 and \#2 (Figure S3). These results demonstrate the importance of the experimental design in the analysis of in vitro models, especially when a large number of samples have to be analyzed and different culture media batches would be expected to be used. In the present study, each batch was used to culture more than one cell line and this design allowed the identification of an anomalous batch (Figures S2 and S3). There is evidence in the literature showing that the cell secretome can be affected by the culture media composition. ${ }^{42}$ 


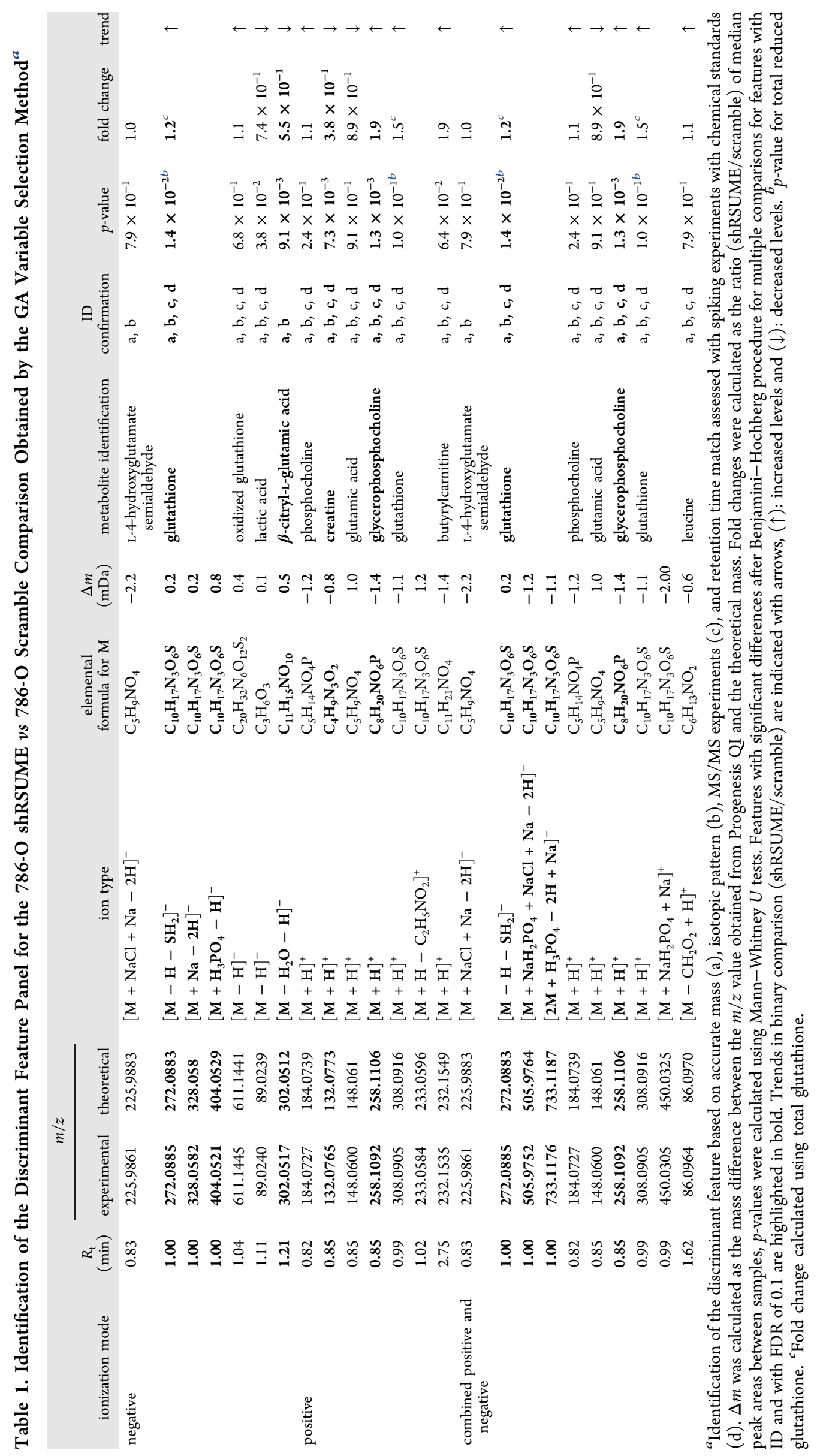


(A)

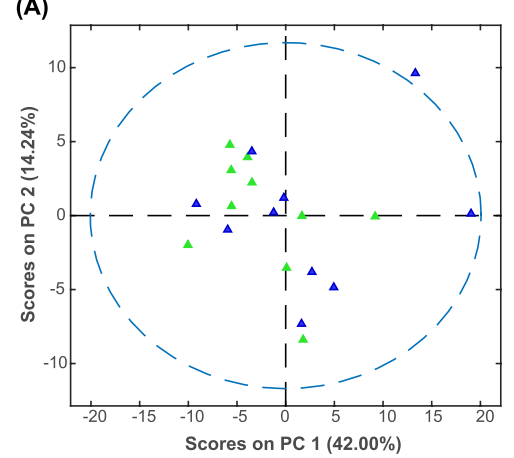

(D)

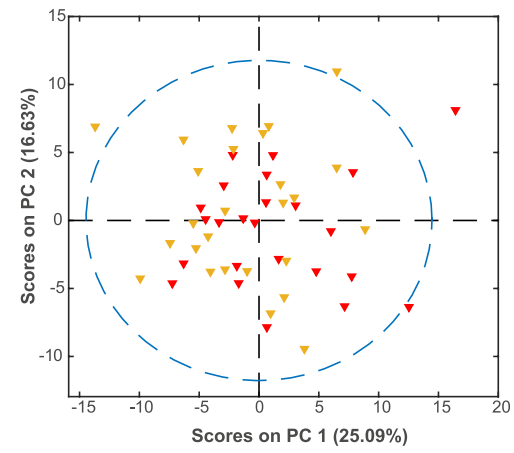

(B)

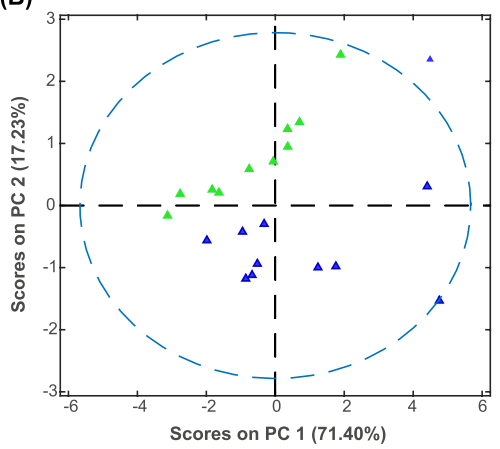

(E)

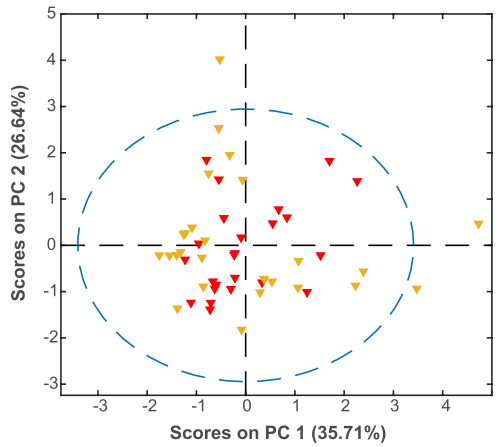

(C)

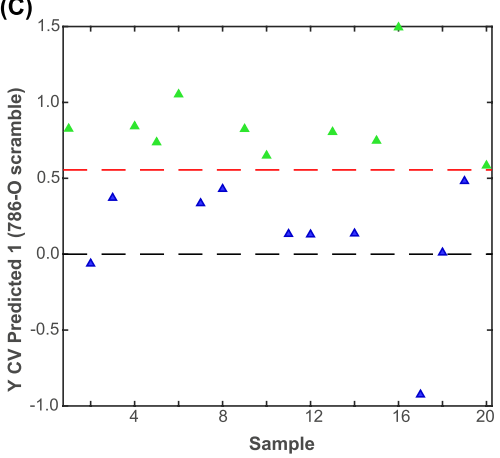

(F)

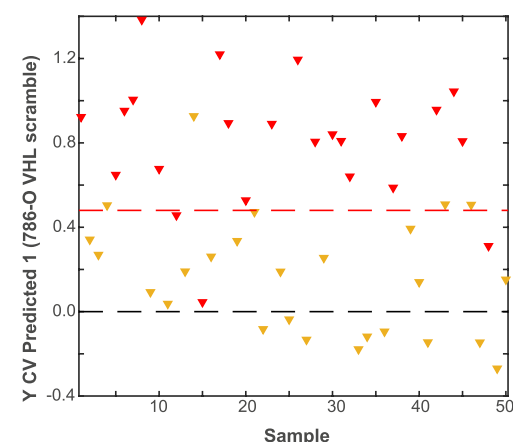

Figure 3. Sample classification. (A) PCA score plot using the set of 128 spectral features (positive ionization mode) for the endometabolome analysis of cell lines 786-O shRSUME (blue up triangles, $n=10$ ) and 786-O scramble (green up triangles, $n=10$ ) with a model that consisted of four PCs with $74.88 \%$ total captured variance. (B) PCA score plot using the set of six identified discriminant metabolites (positive ionization mode) for 786-O shRSUME (blue up triangles, $n=10$ ) and 786-O scramble (green up triangles, $n=10$ ) cell lines with a model that consisted of two PCs with $88.47 \%$ total captured variance. (C) Cross-validated prediction plot from the oPLS-DA analysis model for 786-O shRSUME (blue up triangles, $n=10$ ) and 786-O scramble (green up triangles, $n=10$ ) cell lines using six identified metabolites obtained from the GA variable selection. This oPLS-DA model resulted in 100\% cross-validated accuracy. (D) PCA score plot using the set of 128 spectral features (positive ionization mode) for cell lines 786-O VHL shRSUME (yellow down triangles, $n=26$ ) and 786-O VHL scramble (red down triangles, $n=24$ ) with a model that consisted of five PCs with $66.97 \%$ total captured variance. (E) PCA score plot using the set of five identified discriminant metabolites (positive ionization mode) for 786-O VHL shRSUME (yellow down triangles, $n=26$ ) and 786-O VHL scramble (red down triangles, $n=24$ ) cell lines with a model that consisted of two PCs with $66.45 \%$ total captured variance. (F) Cross-validated prediction plot from the oPLS-DA analysis model for 786-O VHL shRSUME (yellow down triangles, $n=26$ ) and 786-O VHL scramble (red down triangles, $n=24$ ) cell lines using five identified metabolites obtained from the GA variable selection. This oPLS-DA model resulted in $86 \%$ cross-validated accuracy.

To our knowledge, the confounding effect of the culture media in the endometabolome profile has not been reported up to date but should be considered in metabolic fingerprinting studies. These results showcase the importance of the experimental design and QC practices to ensure high-quality data in untargeted metabolomics studies. ${ }^{23,43}$ After removing the data corresponding to the outlier media batch, unsupervised and supervised multivariate analyses were conducted for all data sets (positive, negative, and combined positive and negative ion modes) to investigate the effect of RSUME expression on both types of RCC cells.

Figure $3 \mathrm{~A}$ shows the PCA score plot using the curated set of 128 spectral features obtained in the positive ion mode for cell lines 786-O shRSUME and 786-O scramble. The model consisted of four PCs with $74.88 \%$ total captured variance and did not provide sample class separation or any type of sample clustering. Therefore, sample discrimination was further attempted by means of oPLS-DA coupled to a GA variable selection method, which yielded 19 discriminant metabolic features. Of this reduced feature panel, six identified discriminant metabolites allowed investigating the effect of RSUME expression on 786-O cells (Table 1). The PCA model built with these six identified discriminant metabolites captured $88.47 \%$ of total variance, and the score plot exhibited good sample class separation (Figure 3B). The cross-validated prediction plot for the oPLS-DA model built using these six identified and confirmed discriminant metabolites yielded $100 \%$ classification accuracy and consisted of two latent variables that interpreted $88.39 \%$ and $74.95 \%$ variance from the X-block (feature peak areas) and Y-block (class membership), respectively (Figure 3C). A similar analysis was performed for 786-O clones expressing the gene encoding for the VHL protein. Figure 3D shows the PCA score plot using the set of 128 spectral features from a model that consisted of five PCs with $66.97 \%$ total captured variance, with no evidence of sample clustering. A PCA model built with the 5 identified discriminant metabolites (Table 2) belonging to the 15 discriminant feature panel selected by the GA process yielded a similar total captured variance $(66.45 \%)$ as the model built with the whole feature set but did not exhibit sample class discrimination either (Figure 3E). However, the crossvalidated prediction plot using these five identified and confirmed discriminant metabolites yielded a classification accuracy of $86 \%$ with a two-latent variable model that interpreted $31.14 \%$ and $70.20 \%$ variance from the $\mathrm{X}$ - and $\mathrm{Y}$ blocks, respectively (Figure $3 \mathrm{~F}$ ).

Analogous analyses were performed for these binary comparisons using the data sets obtained from the negative 


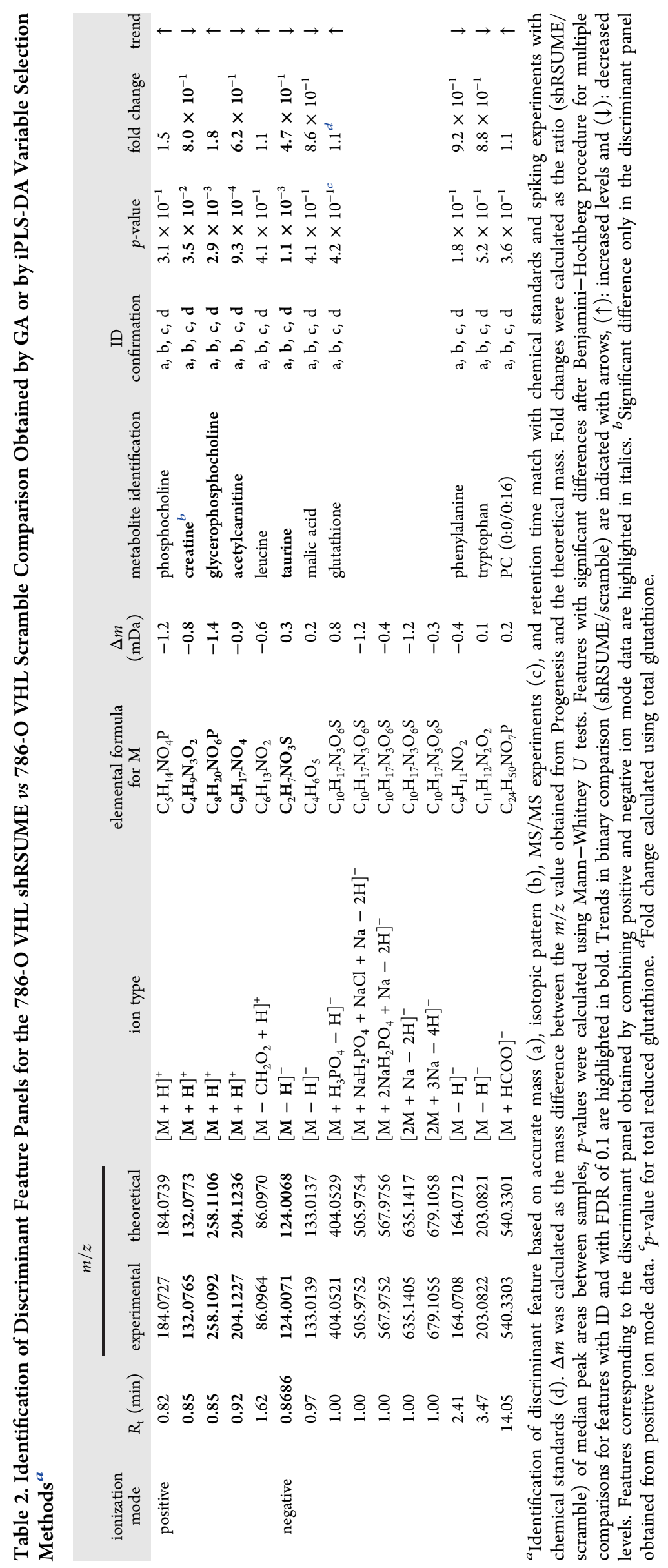


ion mode and combined negative and positive ion modes. Table S3 compares the performance of PCA and oPLS-DA models and shows that discriminant feature panels selected by GA or iPLS-DA methods, as well as reduced panels, composed of identified and confirmed metabolites yielded better classification performance than the initial sets of 128,56 , and 184 features from positive, negative, and a combination of positive and negative data sets, respectively. The only reduced panel that exhibited limited discriminant power compared to the original data set corresponded to the panel with seven identified metabolites from the negative ion mode data for the 786-O VHL shRSUME versus 786-O VHL scramble comparison. Score plots from PCA models built with the original feature sets and with the panels of identified metabolites for negative ion mode data, and for combined positive and negative ion mode data are illustrated in Figures S23 and S24, respectively.

\section{Identification of Discriminant Metabolites}

Metabolite identification was attempted for 75 features resulting from a total of six discriminant feature panels corresponding to the two binary comparisons 786-O shRSUME versus 786-O scramble and 786-O VHL shRSUME versus 786-O VHL scramble (see the Data Analysis and Metabolite Identification Procedure). Because additional 11 features were highly correlated with some of the discriminant features in the selected panels, metabolite identification was also attempted for them, leading to a total of 86 features.

An example of metabolite identification is illustrated in Figure 2 for glutathione and butyrylcarnitine. First, extracted ion chromatograms and the corresponding mass spectra were obtained for each feature in intrastudy QC samples. According to the exact mass of the detected adduct ions or suggested elemental formulae, possible candidates were searched in the $\mathrm{HMDB}^{37}{ }^{37}$ Metlin, ${ }^{38}$ and LIPID MAPS $^{39}$ databases. Subsequently, fragmentation patterns from tandem MS experiments were compared to the MS/MS spectra obtained from HMDB, ${ }^{37}$ Metlin, ${ }^{38}$ and LIPID MAPS ${ }^{39}$ databases or were manually interpreted (Figures S25 and S26). To confirm tentative candidate molecules, retention time and fragmentation pattern matching between samples and chemical standards were used as final identification criteria. Chemical standard analysis also contributed to discard nonmatches with putatively identified molecules, leaving some unidentified features (Tables S4 and S5). Limitations associated with the lack of identification were due to (i) coelution with compounds of similar molecular weight as targets, interfering in the quadrupole selection process of the precursor ion and providing product ion overlap in tandem MS spectra, (ii) insufficient precursor ion intensity for conducting MS/MS experiments, or (iii) limited information in metabolite databases.

Correlation analysis and retention time difference suggested the presence of several ions corresponding to the same metabolite due to in-source fragmentation and/or formation of different adducts. ${ }^{44}$ Out of 86 features, 5 were identified and confirmed as in-source fragment ions, as illustrated in Figure 2, for glutathione. For all cases, the in-source-generated fragments exhibited a Spearman correlation coefficient $>0.87$ with their corresponding precursor ions detected as $[\mathrm{M}+\mathrm{H}]^{+}$or $[\mathrm{M}-\mathrm{H}]^{-}$ionic species. The identification of these ionic species was confirmed by chemical standard matching through MS and MS/MS experiments conducted at low energy ${ }^{44}$
(Figures 2, S19-S22). Therefore, features originally extracted as independent ions by Progenesis QI, once identified, were manually grouped and expressed as total $M$ for further statistical analysis. Similarly, several adduct ions with sodium and formic acid as those identified for glutathione, that is, $[\mathrm{M}$ $+\mathrm{Na}-2 \mathrm{H}]^{-},\left[\mathrm{M}+\mathrm{CHO}_{2} \mathrm{Na}+\mathrm{Na}-2 \mathrm{H}\right]^{-},[2 \mathrm{M}+\mathrm{Na}-$ $2 \mathrm{H}]^{-},[2 \mathrm{M}+3 \mathrm{Na}-4 \mathrm{H}]^{-}$, and $[\mathrm{M}-2 \mathrm{H}+3 \mathrm{Na}]^{+}$(Figures S6, S7, S11, S14 and S17), were incorrectly assigned by the software as features from different molecules. Glutathione also generated adduct ions with phosphoric acid and sodium, including $\left[\mathrm{M}+\mathrm{H}_{3} \mathrm{PO}_{4}+\mathrm{Na}-2 \mathrm{H}\right]^{-},\left[\mathrm{M}+\mathrm{H}_{3} \mathrm{PO}_{4}-\mathrm{H}\right]^{-},[\mathrm{M}$ $\left.+\mathrm{NaH}_{2} \mathrm{PO}_{4}+\mathrm{Na}-2 \mathrm{H}\right]^{-},\left[2 \mathrm{M}+\mathrm{H}_{3} \mathrm{PO}_{4}-\mathrm{H}\right]^{-},[\mathrm{M}+$ $\left.\mathrm{NaH}_{2} \mathrm{PO}_{4}+\mathrm{Na}\right]^{+},\left[\mathrm{M}+2 \mathrm{NaH}_{2} \mathrm{PO}_{4}+\mathrm{Na}-2 \mathrm{H}\right]^{-},[\mathrm{M}+$ $\left.\mathrm{NaH}_{2} \mathrm{PO}_{4}+\mathrm{NaCl}+\mathrm{Na}-2 \mathrm{H}\right]^{-},\left[2 \mathrm{M}+\mathrm{H}_{3} \mathrm{PO}_{4}+\mathrm{Na}-2 \mathrm{H}\right]^{-}$, and $\left[2 \mathrm{M}+\mathrm{H}_{3} \mathrm{PO}_{4}+2 \mathrm{Na}-3 \mathrm{H}\right]^{-}$(Figures S4, S5, S8-S13, S18). The large number of adduct species detected for glutathione is not only related to matrix effects but also associated with the large intracellular levels expected for this compound in the $\mathrm{mM}$ range. ${ }^{45}$

The identified ionic species highlight that the chemical composition of the culture media as well as the methods used for washing and quenching the cell metabolism can drastically influence the detected endometabolome species and favor the formation of nonspecific ion pairing in ESI. These are additional challenges associated with the identification pipeline of metabolic fingerprinting and footprinting studies that need to be accounted for when identifying features generated from in vitro models. In the present work, strategies for accurate identification also included spiking experiments on samples and process blanks, as well as accounting for sodium-assisted inductive cleavage in collision-induced fragmentation mechanisms in tandem MS experiments ${ }^{46}$ (Figures S4-S8, S10, S11, S13-S15, S17, S18).

For taurine, malic acid, oxidized glutathione, lactic acid, phenylalanine, and tryptophan, only the $[\mathrm{M}-\mathrm{H}]^{-}$ion was detected (Figures S27-S32). Similarly, for phosphocholine, creatine, glutamic acid, glycerophosphocholine, butyrylcarnitine, and $o$-acetylcarnitine, only the $[\mathrm{M}+\mathrm{H}]^{+}$ion was detected (Figures 2G-I and S33-S37). In these cases, spiked intrastudy QC samples or pooled samples and chemical standard solutions provided identical retention time values. L-4Hydroxyglutamate semialdehyde and $\beta$-citryl-L-glutamic acid were detected as $[\mathrm{M}-\mathrm{H}]^{-}$and $\left[\mathrm{M}-\mathrm{H}_{2} \mathrm{O}-\mathrm{H}\right]^{-}$, respectively. These compounds were identified with a confidence level of $2^{26,27}$ (Figures S25 and S26) because no chemical standards were used for comparison (Table 1).

The high chromatographic resolution of the analytical method allowed the correct identification of different isomers such as butyrylcarnitine and isobutyrylcarnitine (Figure $2 \mathrm{G}$ ), leucine and isoleucine (Figure S22), and lysophosphatidylcholine $(0: 0 / 16: 0)$ and lysophosphatidylcholine (16:0/0:0) (Figure S38) because they exhibited different retention time values. Overall, 37 of the 86 metabolic features were successfully identified by MS and MS/MS experiments, while 35 out of 37 were further chromatographically confirmed by chemical standards with MSI level 1 (Table 1, 2).

\section{RSUME Effect on 786-O Cells with and without the VHL} Gene

Tables 1 and 2 show the identified metabolites with their confidence levels from the different discriminant panels obtained for the binary comparisons of 786-O shRSUME versus 786-O scramble cells and 786-O VHL shRSUME versus 
(A)

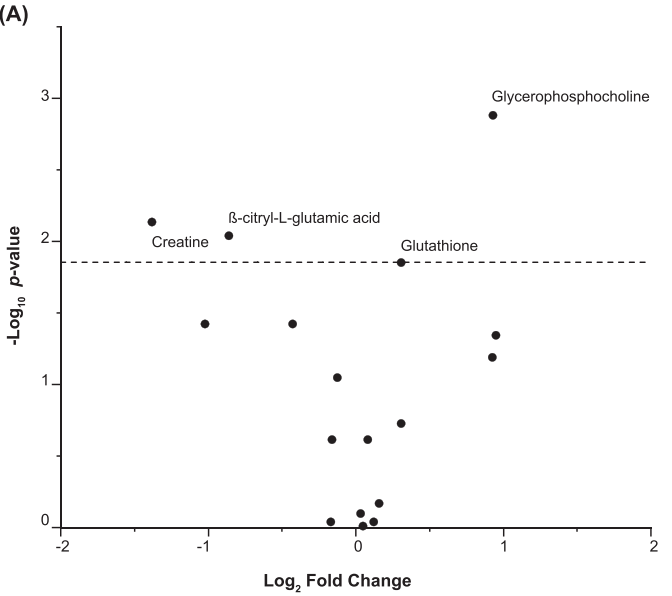

(B)

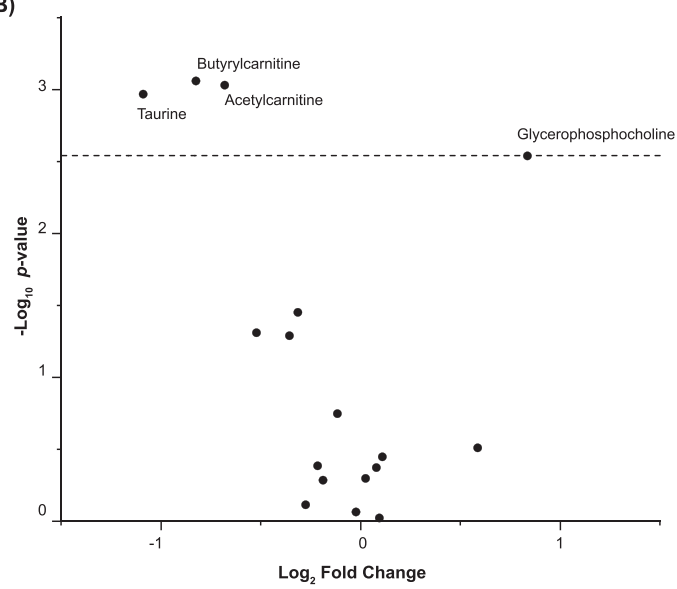

Figure 4. Volcano plots. (A) Out of 17 identified metabolites from negative and positive ion mode data, glycerophosphocholine, $\beta$-citryl-L-glutamic acid, creatine, and glutathione exhibited significant differential abundance (absolute fold change calculated as median ratio, FDR $=0.10, p$-value $=$ 0.014 ) for the binary comparison of 786-O shRSUME $v s 786-\mathrm{O}$ scramble. (B) Out of 17 identified metabolites from negative and positive ion mode data, butyrylcarnitine, acetylcarnitine, taurine, and glycerophosphocholine exhibited significant differential abundance (absolute fold change calculated as median ratio, $\mathrm{FDR}=0.10, p$-value $=0.0029)$ for the binary comparison of 786-O VHL shRSUME $v$ s 786-O VHL scramble. MannWhitney $U$ tests were used to calculate the statistical significance, and $p$-values were corrected using the Benjamini-Hochberg procedure.
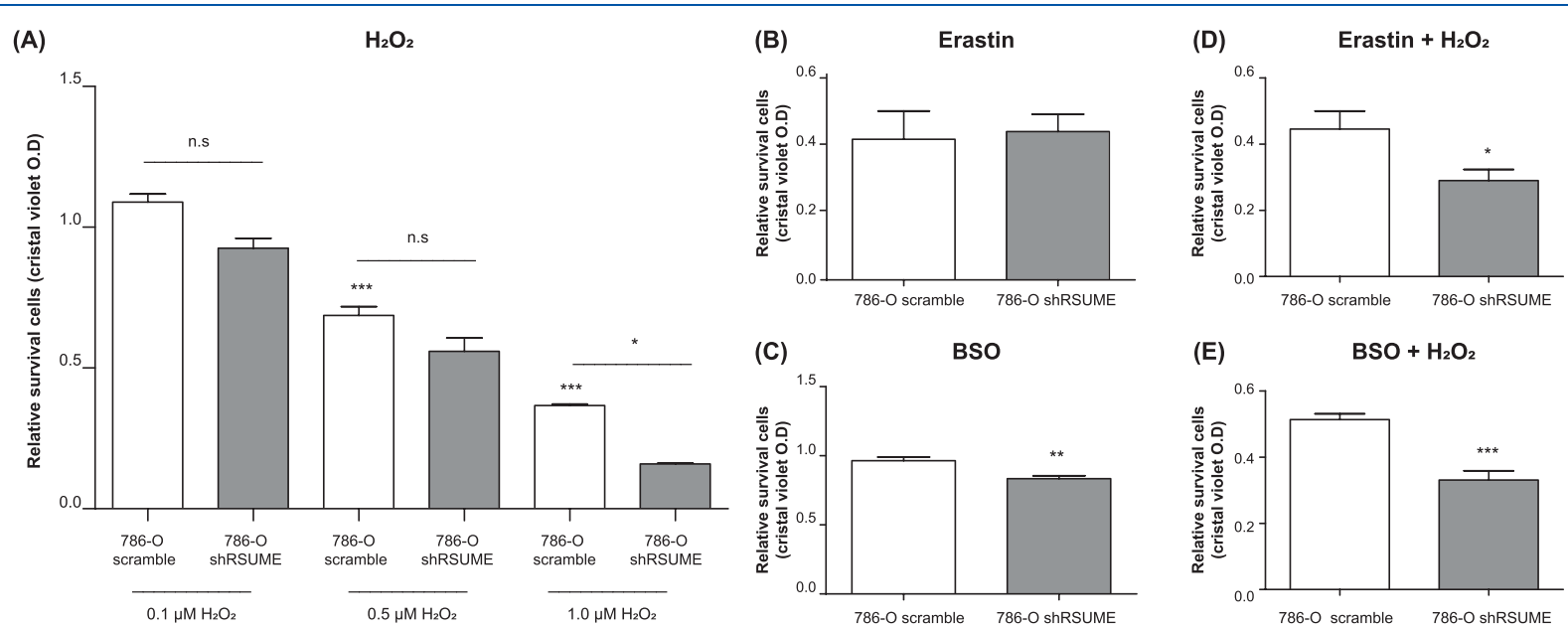

Figure 5. Effect of RSUME on the viability of RCC VHL-deficient cells under oxidative stress and glutathione depletion. (A-E) Crystal violet assay performed to evaluate the cell viability in $786-\mathrm{O}$ cells under $(\mathrm{A}) \mathrm{H}_{2} \mathrm{O}_{2}$ treatment $(0.1,0.5$, and $1 \mu \mathrm{M})$. Data are shown as mean \pm standard error of the mean (SEM). One-way ANOVA and Tukey's test was used to calculate the statistical significance. $* * * p<0.001$ compared to scramble $\left(\mathrm{H}_{2} \mathrm{O}_{2}\right.$, $0.1 \mu \mathrm{M})$ and ${ }^{\#} p<0.05$ compared to scramble $\left(\mathrm{H}_{2} \mathrm{O}_{2}, 1.0 \mu \mathrm{M}\right) ; n=3$; (B) cystine/glutamate transporter inhibitor (erastin, $\left.10 \mu \mathrm{M}\right)$ treatment; (C) $\gamma$-glutamylcystine synthetase inhibitor $(\mathrm{BSO}, 1 \mathrm{mM}$ ) treatment; (D) cysteine/glutamate transporter inhibitor (erastin, $10 \mu \mathrm{M}$ ) plus oxidative stimulus $\left(\mathrm{H}_{2} \mathrm{O}_{2}, 0.5 \mu \mathrm{M}\right)$ treatment; and (E) $\gamma$-glutamylcysteine synthetase inhibitor (BSO, $\left.1 \mathrm{mM}\right)$ plus oxidative stimulus $\left(\mathrm{H}_{2} \mathrm{O}_{2} 0.5 \mu \mathrm{M}\right)$ treatment. Data are shown as mean \pm SEM. An unpaired $t$-test was used to calculate the statistical significance: $* p<0.05$, ** $p<0.01$, and $* * * p<$ $0.001 ; n=3$.

786-O VHL scramble cells, respectively. In addition, volcano plots were built based on the identified metabolites from the different discriminant panels (Figures 4, S39 and S40). Overall, results indicate that the RSUME expression significantly modified the levels of glycerophosphocholine and creatine independently of the presence of VHL. Glutathione and phosphocholine levels were also affected by RSUME expression, with glutathione concentration exhibiting a significant increase in 786-O cells silenced for endogenous RSUME. Conversely, levels of $\beta$-citryl-L-glutamic acid were significantly increased by RSUME expression in 786-O cells. In the presence of VHL, cells expressing RSUME exhibited significantly increased levels of butyrylcarnitine, acetylcarnitine, and taurine. Therefore, the UPLC-MS-based fingerprinting data showed that the discriminant metabolic profiles were associated with both RSUME expression and VHL status. Some identified discriminant metabolites have been previously linked to relevant alterations in ccRCC progression ${ }^{47-53}$ and help to unveil RSUME participation in this type of cancer. ${ }^{8,17}$ Based on the discriminant metabolites found in the study, two of the main identified altered pathways associated with RSUME expression in 786-O and 786-O VHL cells involved the cell antioxidant defense system and the lipid metabolism. Therefore, additional biological experiments and bioinformatics analyses were conducted for glutathione and carnitines, respectively, to further assess the impact of the metabolic fingerprinting results.

\section{Glutathione and the Antioxidant Defense System}

Glutathione was identified as a discriminant metabolite between RSUME statuses in ccRCC cells defective for VHL. 
(A)

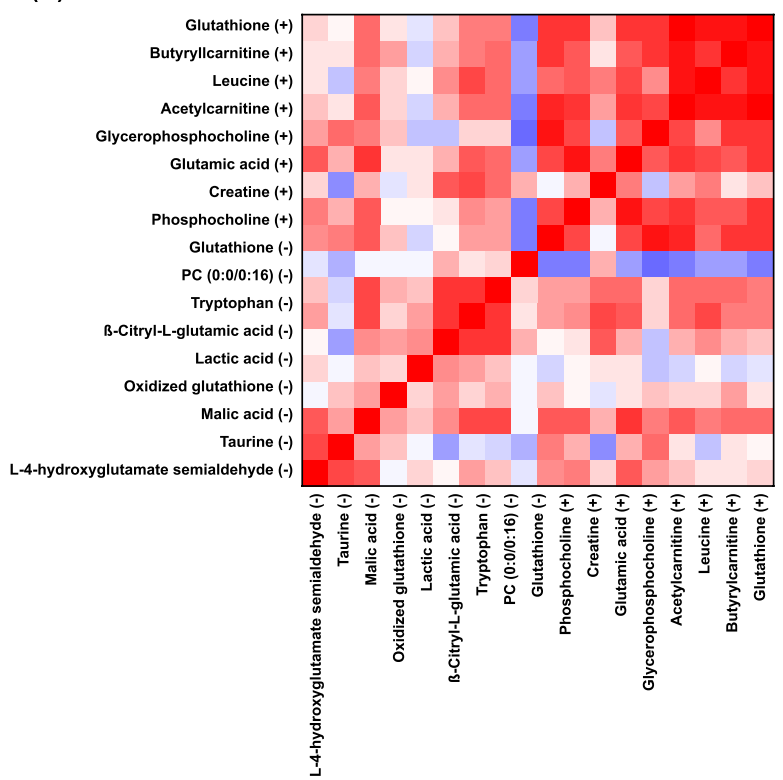

(B)

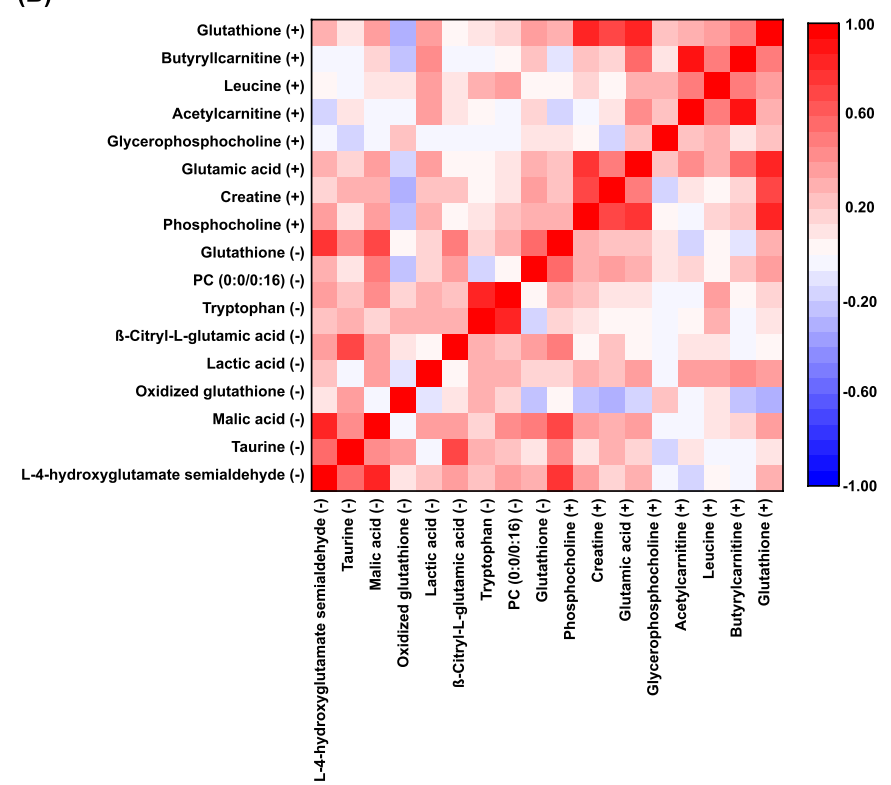

Figure 6. Heat maps. Pairwise correlation values of 18 identified metabolites for the binary comparison of 786-O shRSUME vs 786-O scramble cells (panel A) and for the binary comparison of 786-O VHL shRSUME vs 786-O VHL scramble cells (panel B). Spearman's correlation coefficients were calculated. Lysophosphatidylcholine (0:0/16:0) (PC (0:0/16:0)).

RSUME-silenced 786-O cells showed a significant increase in glutathione levels, with a fold change of 1.2 compared to control cells (Table 1). It has been previously shown that the highly energetic demand of proliferating cancer cells leads to reactive oxygen species (ROS) accumulation ${ }^{54,55}$ and induces the activation of antioxidant pathways. ${ }^{56}$ Glutathione is the main ROS scavenger in cells ${ }^{55}$ and becomes relevant in ccRCC because superoxide dismutase, which is an antioxidant enzyme, is inhibited by HIF upregulation ${ }^{57}$ driven by VHL loss. Glutathione has been associated with RCC tumor progres$\operatorname{sion}^{55}$ and has exhibited a strong classification value for RCC patient outcome. ${ }^{52}$ The ROS threshold that determines a proproliferative effect or cell death induction mediated by an excessive and irreversible damage is a key factor to understand the significance of RSUME-mediated glutathione levels. ${ }^{54,58}$

To gain more insights into how glutathione increase in 786O shRSUME cells regulates cell viability, the cell lines were exposed to an oxidative challenge (Figure 5). Reduced 786-O cell viability was evidenced for increased $\mathrm{H}_{2} \mathrm{O}_{2}$ concentrations, with 786-O shRSUME cells being more affected than scramble cells to the oxidative challenge (Figure 5A). Higher glutathione levels found in 786-O shRSUME cells compared to scramble cells were still not enough to counter ROS accumulation, suggesting that other affected antioxidant pathways may be involved. To further reveal differences among these cell lines regarding the role of the glutathione pathway in preserving the redox balance, cells were treated with two glutathione synthesis inhibitors, that is, $\gamma$-glutamylcysteine synthetase inhibitor (BSO) and cystine glutamate antiporter inhibitor (erastin). The latter was unable to significantly modify the cell viability between VHL-deficient cell lines (Figure 5B). In contrast, the cell viability was significantly reduced in RSUME-silenced cells compared to control cells under BSO treatment (Figure 5C), suggesting that shRSUME cells use the glutathione antioxidant system to counter higher ROS levels. The synergistic effect of oxidative stimuli and glutathione depletion was also analyzed with the intermediate tested concentration for $\mathrm{H}_{2} \mathrm{O}_{2}(0.5 \mu \mathrm{M})$.
The cell viability was significantly decreased in shRSUME cells compared to control cells when they were exposed to an oxidative insult and glutathione synthesis inhibition under both synergistic treatments, that is, BSO and erastin (Figure 5D,E). The fact that erastin alone failed to induce viability changes (Figure 5B) in these cells with different levels of glutathione was surprising given that cysteine has been proposed as to be essential for the synthesis of this metabolite in cancer. ${ }^{47,59}$ Possibly, cysteine is generated through the cystathioninemethionine cycle, which is also linked to choline derivatives. ${ }^{60}$ In line with this, we also found significantly increased levels of glycerophosphocholine in 786-O shRSUME cells compared to 786-O scramble cells with a fold change of 1.9 (Table 1). Moreover, this metabolite exhibited high correlation with glutathione, acetylcarnitine, and butyrylcarnitine for the binary comparison of 786-O shRSUME versus 786-O scramble cells (Figure 6A), suggesting that choline derivatives would participate in the folate/methionine Q5 cycle, which is important for glutathione synthesis, and can also act as lipid reservoirs for energy production. ${ }^{60}$ Altogether these results show that metabolic changes derived by RSUME-silencing support an alteration in the redox balance in 786-O shRSUME cells.

The induction of ROS production has been a widely accepted strategy for the treatment of ccRCC patients, but it is currently used as a combined therapy given the high drug resistance. ${ }^{61-63}$ It has been demonstrated that VHL expression decreases BSO and erastin sensitivity in ccRCC cells. ${ }^{47}$ Therefore, a viability experiment was also conducted for VHL re-expressing cells (Figure S41). Slight increments of $\mathrm{H}_{2} \mathrm{O}_{2}$ concentration significantly reduced the viability of 786$\mathrm{O}$ VHL cells, but no differences were observed regarding RSUME expression (Figure S41A). Similarly, no difference was evidenced under BSO (Figure S41B) or erastin treatment alone (Figure S41C) in RSUME-silenced cells compared to control cells. When the synergistic effect of oxidative stimuli and glutathione depletion was analyzed for VHL re-expressing 
(A)
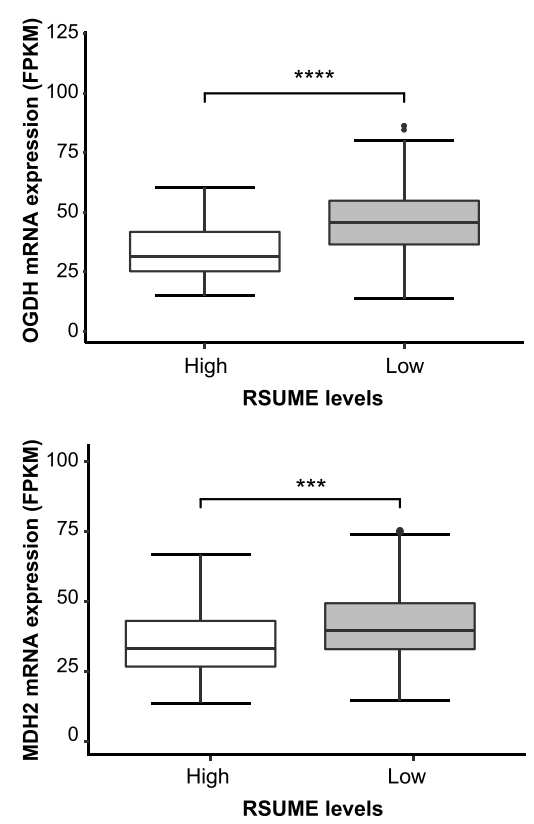

(B)

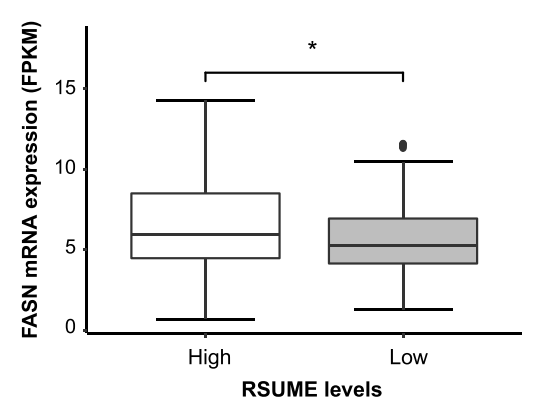

(C)

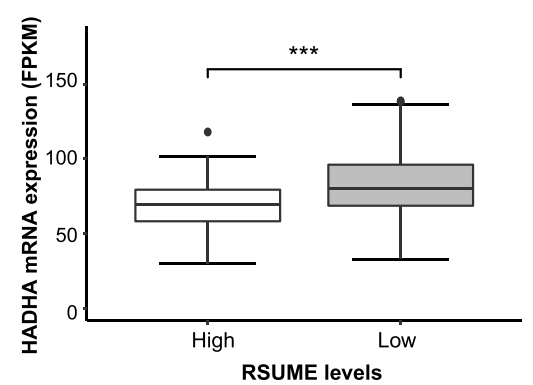

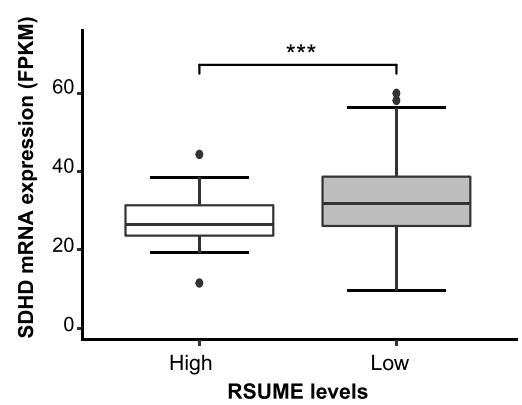
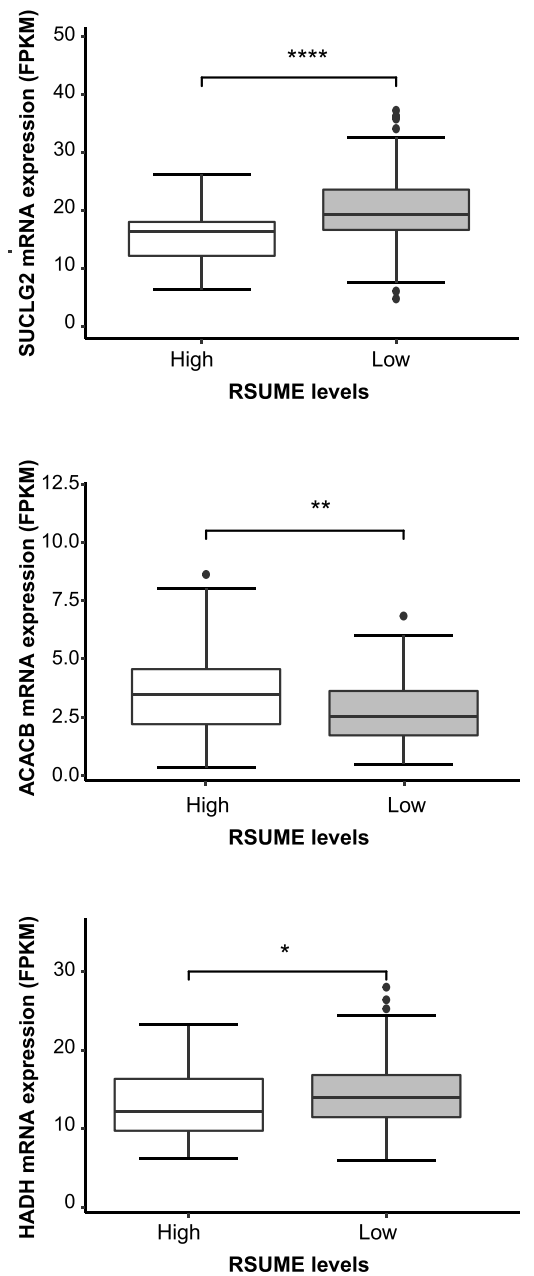

Figure 7. Evaluation of RSUME effect on the mRNA expression of enzymes related to the fatty acid metabolism and the TCA cycle in VHLpositive RCC patients. Box plots show mRNA expression of genes involved in (A) TCA cycle, (B) FAS, or (C) FAO pathways. Comparisons were made between high $(n=44)$ and low $(n=171)$ RSUME expression within VHL-positive patients $(n=215)$. Box plots were constructed from the minimum value, the first quartile, the median, the third quartile, and the maximum value of each set of data. Mann-Whitney $U$ tests were used to calculate the statistical significance: $*_{p}<0.05, * * p<0.01, * * * p<0.001$, and $* * * *_{p}<0.0001$.

RCC cell lines, the cell viability was significantly decreased for both BSO (Figure S41D) and erastin (Figure S41E), compared with RSUME-silenced cells. Interestingly, VHL re-expressing cells exhibited an opposite response than VHL-deficient cells to the synergistic effect mediated by stimuli cotreatment (Figure $5 \mathrm{D}, \mathrm{E})$. The differences observed regarding viability associated with RSUME expression may be related to oxidative phosphorylation promoted by VHL. ${ }^{47,64}$ In agreement with previous works, ${ }^{47}$ only VHL-deficient cell lines treated with BSO exhibited differential decreased viabilities correlated with glutathione levels. The fact that $786-\mathrm{O}$ scramble cells presented lower response to BSO treatment compared with shRSUME cells opens the question to evaluate RSUME levels as a predictive biomarker for ROS-targeted therapies.

\section{Carnitines and the Lipid Metabolism}

The metabolic fingerprinting results indicate that RSUME expression significantly increased the levels of acetylcarnitine and butyrylcarnitine in 786-O cells re-expressing VHL (Table 2 and Figure 4). The levels of these two compounds and other carnitine derivatives were found to be increased in neoplastic renal tissue and urine from RCC patients ${ }^{60}$ and have correlated with kidney cancer grade, ${ }^{49,65,66}$ suggesting a role in tumor 
progression. Accordingly, increased RSUME expression was found in stage IV ccRCC tumors. ${ }^{17}$ Previous studies ${ }^{49,52,66}$ have shown that TCA cycle enzymes are downregulated at transcriptional and protein levels in ccRCC tissue compared to nontumor tissue. Therefore, a bioinformatics approach was conducted to evaluate the potential TCA cycle downregulation coupled to RSUME expression to understand the significance of the elevated acetylcarnitine levels found in 786-O VHL scramble cells compared to RSUME-silenced cells. The expression of TCA cycle enzymes was assessed using the TCGA Kidney Renal Clear Cell Carcinoma (TCGA-KIRC) data collection, and levels of RSUME expression were compared within VHL-positive ccRCC patients (Figure 7A). This analysis showed that patients with higher levels of RSUME expression exhibited lower levels of TCA cycle enzymes.

Considering that acetylcarnitine is transported to the cytosol, providing acetyl groups for the synthesis of fatty acids, we also evaluated if RSUME affects enzymes involved in FAS. Figure $7 \mathrm{~B}$ shows that fatty acid synthase (FASN), the central enzyme in de novo lipogenesis, and acetyl-CoA carboxylase 2, which catalyzes the rate-limiting step in FAS, were upregulated in VHL-positive ccRCC patients with higher RSUME levels. This increase in lipogenesis has been previously associated with tumor progression, ${ }^{67,68}$ supporting the protumor role of RSUME.

Given that FAO is an acetyl-CoA-supplying process serving as a source of TCA cycle and that FAO and FAS are antagonistic, ${ }^{69-71}$ an additional bioinformatics analysis was conducted to evaluate how RSUME levels affect enzymes involved in FAO in VHL-positive ccRCC patients. Figure 7C shows that both (S)-3-hydroxyacyl-CoA dehydrogenase $(\mathrm{HADH})$, essential in the mitochondrial $\beta$-oxidation of short-chain fatty acids, and $\mathrm{HADH}$ trifunctional multienzyme complex subunit alpha (HADHA), involved in medium-chainlength fatty acid oxidation, were downregulated by RSUME. This $\beta$-oxidation enzyme modulation has been previously proven by a proteomics analysis ${ }^{66,67}$ when comparing normal kidney with different ccRCC tumor grades. This information supports that increased levels of acetylcarnitine and butyrylcarnitine in 786-O VHL scramble cells may be associated with upregulation of FAS and inhibition of TCA and FAO pathways. These metabolic changes resemble protumor features of ccRCC tumors. ${ }^{66-68}$ In addition, downregulation of TCA cycle genes and upregulation of genes in the pentose phosphate pathway and FAS have correlated with ccRCC progression. ${ }^{52,67,72-75}$ In line with this, reduced expression of TCA cycle enzymes (OGDH, SDHD, MDH2, and SUCLG2), higher expression of a key FAS enzyme (FASN), and reduced expression of FAO enzymes (HADHA and $\mathrm{HADH}$ ) were associated with poor ccRCC outcome (Figure S42).

Significant changes in amino acid metabolism have been reported in VHL re-expressing RCC cells compared with VHLdeficient cells. ${ }^{65}$ Accordingly, several amino acids were identified as discriminant metabolites between 786-O VHL shRSUME and 786-O VHL scramble cells, such as leucine, phenylalanine, tryptophan, and taurine, the latter exhibiting a significant increase in scramble cells (Table 2). Moreover, a high correlation was found between glutathione and glutamic acid, creatine, and phosphocholine (Figure 6). In accordance, these three compounds have been reported to be involved in the glutathione synthesis pathway. ${ }^{60}$ Significantly larger levels of creatine were detected in both 786-O scramble and 786-O
VHL scramble cells in comparison with their corresponding shRSUME cells, which may contribute to glutathione increment in these cells through the folate/methionine cycle. ${ }^{60}$ Overall, these results show that RSUME is involved in the downregulation of the antioxidant defense system in the absence of VHL, whereas in the presence of VHL, it acts in rerouting energy related-pathways, negatively modulating the lipid utilization and positively modulating the FAS, which may promote deposition in droplets (Figure 8).

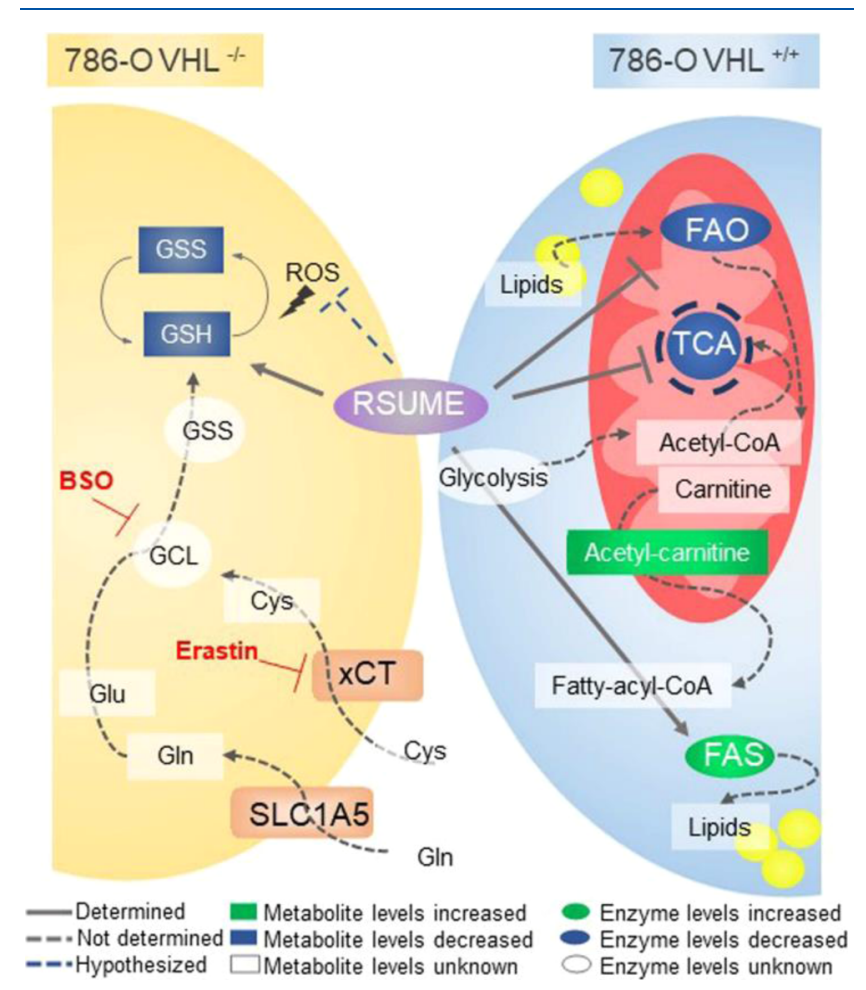

Figure 8. Schematic diagram of VHL-RSUME regulation of metabolic changes in ccRCC cells: TCA cycle and fatty acid oxidation (FAO) down modulation, FAS upregulation induced by RSUME expression in 786-O ccRCC cells expressing VHL, and reduction of glutathione levels induced by RSUME expression in 786-O VHL-deficient ccRCC cells.

\section{CONCLUSIONS}

Results from UPLC-QTOF-MS-based metabolic fingerprinting experiments of human ccRCC cell lines supported by biological experiments and bioinformatics analyses provided here a combined strategy to evaluate the role of RSUME in cell metabolism. We optimized a protocol for harvesting, quenching, extracting, and profiling the endometabolome derived from 786-O cells. Multivariate models yielded discriminant metabolic panels that differentiated with high classification accuracy the metabolic profiles based on the RSUME and VHL statuses. Discriminant metabolites identified with MSI level 1 and results from biological experiments and bioinformatics analyses provided clues to understand altered pathways associated with RSUME expression in 786-O and 786-O VHL cells and open the question to evaluate RSUME levels as a predictive biomarker for ROS-targeted therapies response. Overall, our findings indicate a disruption of the cell antioxidant defense system and modulation of fatty acid metabolism depending on the RSUME and VHL statuses and 
provide new opportunities for unveiling the role of RSUME in ccRCC.

\section{ASSOCIATED CONTENT}

\section{Supporting Information}

The Supporting Information is available free of charge at https://pubs.acs.org/doi/10.1021/acs.jproteome.0c00655.

Culture media effect: PCA score plot for endometabolome analysis of cell lines 786-O shRSUME, 786-O scramble, 786-O VHL shRSUME, 786-O VHL scramble, and pooled QC samples using the set of 184 metabolic features; culture media effect in negative, positive, and combined ionization mode data; PCA score plot for endometabolome analysis of cell lines 786-O shRSUME and 786-O scramble; culture media effect in negative, positive, and combined ionization mode data; PCA score plot for endometabolome analysis of cell lines 786O VHL shRSUME and 786-O VHL scramble; parameters and results for GA and iPLS-DA variable selection methods; identification of glutathione as [glutathione $\left.+\mathrm{NaH}_{2} \mathrm{PO}_{4}+\mathrm{Na}-2 \mathrm{H}\right]^{-}$adduct ions; identification of glutathione as a [glutathione $+\mathrm{Na}-$ $2 \mathrm{H}]^{-}$adduct ions; identification of glutathione as [glutathione $\left.+\mathrm{NaH}_{2} \mathrm{PO}_{4}+\mathrm{Na}-2 \mathrm{H}\right]^{-}$adduct ions; identification of glutathione as [glutathione $+\mathrm{CHO}_{2} \mathrm{Na}$ $+\mathrm{Na}-2 \mathrm{H}]^{-}$adduct ions; identification of glutathione as [glutathione $\left.+\mathrm{H}_{3} \mathrm{PO}_{4}+\mathrm{Na}-2 \mathrm{H}\right]^{-}$adduct ions; identification of glutathione as [glutathione $+\mathrm{H}_{3} \mathrm{PO}_{4}-$ $\mathrm{H}]^{-}$adduct ions; identification of glutathione as [glutathione $\left.+\mathrm{NaH}_{2} \mathrm{PO}_{4}+\mathrm{NaCl}+\mathrm{Na}-2 \mathrm{H}\right]^{-}$adduct ions; identification of glutathione as $[2$ glutathione $+\mathrm{Na}$ $-2 \mathrm{H}]^{-}$adduct ions; identification of glutathione as [2 glutathione $\left.+\mathrm{H}_{3} \mathrm{PO}_{4}-2 \mathrm{H}\right]^{-}$adduct ions; identification of glutathione as [2 glutathione $\left.+\mathrm{H}_{3} \mathrm{PO}_{4}+\mathrm{Na}-2 \mathrm{H}\right]^{-}$ adduct ions; identification of glutathione as [2 glutathione $+3 \mathrm{Na}-4 \mathrm{H}]^{-}$adduct ions; identification of glutathione as $\left[2 \text { glutathione }+\mathrm{H}_{3} \mathrm{PO}_{4}+2 \mathrm{Na}-3 \mathrm{H}\right]^{-}$ adduct ions; identification of glutathione as [glutathione $+\mathrm{H}]^{+}$adduct ions; identification of glutathione as [glutathione $-2 \mathrm{H}+3 \mathrm{H}]^{+}$adduct ions; identification of glutathione as [glutathione $\left.+\mathrm{NaH}_{2} \mathrm{PO}_{4}+\mathrm{Na}\right]^{+}$adduct ions; identification of glutathione as [glutathione $\left.\mathrm{C}_{5} \mathrm{H}_{7} \mathrm{NO}_{3}+\mathrm{H}\right]^{+}$adduct ions; identification of glutathione as [glutathione $\left.-\mathrm{H}_{2} \mathrm{O}+\mathrm{H}\right]^{+}$adduct ions; identification of glutathione as [glutathione $-\mathrm{C}_{2} \mathrm{H}_{5} \mathrm{NO}_{2}$ $+\mathrm{H}]^{+}$adduct ions; identification of leucine; matrix raw data curation; results from PCA and oPLS-DA analysis; PCA score plot for cell lines 786-O VHL shRSUME versus 786-O VHL scramble and 786-O shRSUME versus $786-\mathrm{O}$ scramble using the set of 56 features and identified discriminant features (negative ionization mode); PCA score plot for the binary comparisons of 786-O VHL shRSUME versus 786-O VHL scramble cells and 786-O shRSUME versus 786-O scramble cells using the set of 184 features and identified discriminant features (positive and negative ionization modes); identification of L-4-hydroxyglutamate semialdehyde; identification of beta-citryl-L-glutamic acid; nonidentified discriminant features for the 786-O shRSUME versus 786-O scramble cells' binary comparison; nonidentified discriminant features for the $786-\mathrm{O} \mathrm{VHL}$ shRSUME versus 786-O VHL scramble cells' binary comparison; identification of taurine; identification of malic acid; identification of oxidized glutathione; identification of lactic acid; identification of phenylalanine; identification of tryptophan; identification of phosphocholine; identification of creatine; identification of glutamic acid; identification of glycerophosphocholine; identification of $\mathrm{O}$-acetyl-carnitine; identification of lysophosphatidylcholine (0:0/16:0); volcano plot for cell lines 786-O VHL shRSUME versus 786-O VHL scramble and 786-O shRSUME versus786-O scramble using identified metabolites from the negative ionization mode data; volcano plot for cell lines 786-O VHL shRSUME versus 786-O VHL scramble and 786-O shRSUME versus 786-O scramble using identified metabolites from the positive ionization mode data; oxidative stress and glutathione depletion differentially affect ccRCC cell viability regarding RSUME expression; and alterations in fatty acid or oxidation and TCA cycle gene expression correlated with poor survival in ccRCC patients (PDF)

Data Set S1 (XLSX)

\section{AUTHOR INFORMATION}

\section{Corresponding Author}

María Eugenia Monge - Centro de Investigaciones en Bionanociencias (CIBION), Consejo Nacional de Investigaciones Científicas y Ténicas (CONICET), C1425FQD Ciudad de Buenos Aires, Argentina; 10 orcid.org/ 0000-0001-6517-5301; Phone: +54 1148995500 (ext.

5614); Email: maria.monge@cibion.conicet.gov.ar

\section{Authors}

Manuela R. Martinefski - Centro de Investigaciones en Bionanociencias (CIBION), Consejo Nacional de Investigaciones Científicas y Técnicas (CONICET), C1425FQD Ciudad de Buenos Aires, Argentina; Facultad de Farmacia y Bioquimica, Universidad de Buenos Aires, C1113AAD Ciudad de Buenos Aires, Argentina

Belén Elguero - Instituto de Investigaciones en Biomédicas de Buenos Aires (IBioBA-CONICET), Partner Institute of the Max Planck Society, C1425FQD Ciudad de Buenos Aires, Argentina

María Elena Knott - Centro de Investigaciones en Bionanociencias (CIBION), Consejo Nacional de Investigaciones Científicas y Técnicas (CONICET), C1425FQD Ciudad de Buenos Aires, Argentina

David Gonilski - Instituto de Investigaciónes en Biomédicas de Buenos Aires (IBioBA-CONICET), Partner Institute of the Max Planck Society, C1425FQD Ciudad de Buenos Aires, Argentina

Lucas Tedesco - Instituto de Investigaciones en Biomédicas de Buenos Aires (IBioBA-CONICET), Partner Institute of the Max Planck Society, C1425FQD Ciudad de Buenos Aires, Argentina

Juan M. Gurevich Messina - Centro de Investigaciones en Bionanociencias (CIBION), Consejo Nacional de Investigaciones Científicas y Técnicas (CONICET), C1425FQD Ciudad de Buenos Aires, Argentina

Cora Pollak - Instituto de Investigaciones en Biomédicas de Buenos Aires (IBioBA-CONICET), Partner Institute of the Max Planck Society, C1425FQD Ciudad de Buenos Aires, Argentina 
Eduardo Arzt - Instituto de Investigaciónes en Biomédicas de Buenos Aires (IBioBA-CONICET), Partner Institute of the Max Planck Society, C1425FQD Ciudad de Buenos Aires, Argentina; Facultad de Ciencias Exactas y Naturales, Universidad de Buenos Aires, C1428EGA Ciudad de Buenos Aires, Argentina

Complete contact information is available at:

https://pubs.acs.org/10.1021/acs.jproteome.0c00655

\section{Author Contributions}

${ }^{\perp}$ M.R.M. and B.E. contributed equally to this work.

Notes

The authors declare no competing financial interest.

\section{ACKNOWLEDGMENTS}

CONICET (Consejo Nacional de Investigaciones Científicas y Técnicas, Argentina), the National Agency of Scientific and Technological Promotion (PICT-2014-3634 project), the University of Buenos Aires, and FOCEM-Mercosur (COF 03/11) are acknowledged for providing the funding. M.R.M., E.A., and M.E.M. are research staff members from CONICET.

\section{REFERENCES}

(1) Hsieh, J. J.; Purdue, M. P.; Signoretti, S.; Swanton, C.; Albiges, L.; Schmidinger, M.; Heng, D. Y.; Larkin, J.; Ficarra, V. Renal cell carcinoma. Nat. Rev. Dis. Primers 2017, 3, 17009.

(2) Hakimi, A. A.; Pham, C. G.; Hsieh, J. J. A clear picture of renal cell carcinoma. Nat. Genet. 2013, 45, 849.

(3) Pinthus, J. H.; Whelan, K. F.; Gallino, D.; Lu, J.-P.; Rothschild, $\mathrm{N}$. Metabolic features of clear-cell renal cell carcinoma: mechanisms and clinical implications. Can. Urol. Assoc. J. 2011, 5, 274.

(4) Gebhard, R. L.; Clayman, R. V.; Prigge, W. F.; Figenshau, R.; Staley, N. A.; Reesey, C.; Bear, A. Abnormal cholesterol metabolism in renal clear cell carcinoma. J. Lipid Res. 1987, 28, 1177.

(5) Linehan, W. M.; Bratslavsky, G.; Pinto, P. A.; Schmidt, L. S.; Neckers, L.; Bottaro, D. P.; Srinivasan, R. Molecular Diagnosis and Therapy of Kidney Cancer. Annu. Rev. Med. 2010, 61, 329.

(6) Banks, R. E.; Tirukonda, P.; Taylor, C.; Hornigold, N.; Astuti, D.; Cohen, D.; Maher, E. R.; Stanley, A. J.; Harnden, P.; Joyce, A.; Knowles, M.; Selby, P. J. Genetic and Epigenetic Analysis of von Hippel-Lindau (VHL) Gene Alterations and Relationship with Clinical Variables in Sporadic Renal Cancer. Cancer Res. 2006, 66, 2000.

(7) Calzada, M. J.; Esteban, M. A.; Feijoo-Cuaresma, M.; Castellanos, M. C.; Naranjo-Suárez, S.; Temes, E.; Méndez, F.; Yánez-Mo, M.; Ohh, M.; Landázuri, M. O. von Hippel-Lindau Tumor Suppressor Protein Regulates the Assembly of Intercellular Junctions in Renal Cancer Cells through Hypoxia-Inducible Factor-Independent Mechanisms. Cancer Res. 2006, 66, 1553.

(8) Gerez, J. L.; Tedesco, J. J.; Fuertes, M.; Barontini, M.; Silberstein, S.; Wu, Y.; Renner, U.; Páez-Pereda, M.; Holsboer, F.; Stalla, G. K.; Arzt, E. RSUME inhibits VHL and regulates its tumor suppressor function. Oncogene 2015, 34, 4855.

(9) Godinot, C.; de Laplanche, E.; Hervouet, E.; Simonnet, H. Actuality of Warburg's views in our understanding of renal cancer metabolism. J. Bioenerg. Biomembr. 2007, 39, 235.

(10) Carbia-Nagashima, A.; Gerez, J.; Perez-Castro, C.; Paez-Pereda, M.; Silberstein, S.; Stalla, G. K.; Holsboer, F.; Arzt, E. RSUME, a Small RWD-Containing Protein, Enhances SUMO Conjugation and Stabilizes HIF-1 $\alpha$ during Hypoxia. Cell 2007, 131, 309.

(11) Gerez, J.; Fuertes, M.; Tedesco, L.; Silberstein, S.; Sevlever, G.; Paez-Pereda, M.; Holsboer, F.; Turjanski, A. G.; Arzt, E. In silico structural and functional characterization of the RSUME splice variants. PLoS One 2013, 8, No. e57795.
(12) Gerez, J.; Tedesco, L.; Bonfiglio, J. J.; Fuertes, M.; Barontini, M.; Silberstein, S.; Wu, Y.; Renner, U.; Páez-Pereda, M.; Holsboer, F.; Stalla, G. K.; Arzt, E. RSUME inhibits VHL and regulates its tumor suppressor function. Oncogene 2015, 34, 4855.

(13) He, W.; Huang, L.; Shen, X.; Yang, Y.; Wang, D.; Yang, Y.; Zhu, $\mathrm{X}$. Relationship between RSUME and HIF-1 $\alpha$ /VEGF-A with invasion of pituitary adenoma. Gene 2017, 603, 54 .

(14) Shan, B.; Gerez, J.; Haedo, M.; Fuertes, M.; Theodoropoulou, M.; Buchfelder, M.; Losa, M.; Stalla, G. K.; Arzt, E.; Renner, U. RSUME is implicated in HIF-1-induced VEGF-A production in pituitary tumour cells. Endocr-Relat. Cancer 2012, 19, 13.

(15) Wu, Y.; Tedesco, L.; Lucia, K.; Schlitter, A. M.; Garcia, J. M.; Esposito, I.; Auernhammer, C. J.; Theodoropoulou, M.; Arzt, E.; Renner, U.; Stalla, G. K. RSUME is implicated in tumorigenesis and metastasis of pancreatic neuroendocrine tumors. Oncotarget 2016, 7, 57878 .

(16) Ji, C.-X.; Fan, Y.-H.; Xu, F.; Lv, S.-G.; Ye, M.-H.; Wu, M. J.; Zhu, X.-G.; Wu, L. MicroRNA-375 inhibits glioma cell proliferation and migration by downregulating RWDD3 in vitro. Oncol. Rep. 2018, 39,1825 .

(17) Tedesco, L.; Elguero, B.; Pacin, D. G.; Senin, S.; Pollak, C.; Garcia Marchinena, P. A.; Jurado, A. M.; Isola, M.; Labanca, M. J.; Palazzo, M.; Yankilevich, P.; Fuertes, M.; Arzt, E. von Hippel-Lindau mutants in renal cell carcinoma are regulated by increased expression of RSUME. Cell Death Discovery 2019, 10, 266.

(18) Hanahan, D.; Weinberg, R. A. Hallmarks of cancer: the next generation. Cell 2011, 144, 646.

(19) Patti, G. J.; Yanes, O.; Siuzdak, G. Metabolomics: the apogee of the omics trilogy. Nat. Rev. Mol. Cell Biol. 2012, 13, 263.

(20) Liu, J.; Hanavan, P. D.; Kras, K.; Ruiz, Y. W.; Castle, E. P.; Lake, D. F.; Chen, X.; O’Brien, D.; Luo, H.; Robertson, K. D.; Gu, H.; Ho, T. H. Loss of SETD2 Induces a Metabolic Switch in Renal Cell Carcinoma Cell Lines toward Enhanced Oxidative Phosphorylation. J. Proteome Res. 2019, 18, 331.

(21) Cuperlović-Culf, M.; Barnett, D. A.; Culf, A. S.; Chute, I. Cell culture metabolomics: applications and future directions. Drug Discovery Today 2010, 15, 610.

(22) Halama, A. Metabolomics in cell culture-A strategy to study crucial metabolic pathways in cancer development and the response to treatment. Arch. Biochem. Biophys. 2014, 564, 100.

(23) Manzi, M.; Riquelme, G.; Zabalegui, N.; Monge, M. E. Improving diagnosis of genitourinary cancers: Biomarker discovery strategies through mass spectrometry-based metabolomics. J. Pharm. Biomed. Anal. 2020, 178, 112905.

(24) Knott, M. E.; Manzi, M.; Zabalegui, N.; Salazar, M. O.; Puricelli, L. I.; Monge, M. E. Metabolic Footprinting of a Clear Cell Renal Cell Carcinoma in Vitro Model for Human Kidney Cancer Detection. J. Proteome Res. 2018, 17, 3877.

(25) Gnarra, J. R.; Tory, K.; Weng, Y.; Schmidt, L.; Wei, M. H.; Li, H.; Latif, F.; Liu, S.; Chen, F.; Duh, F.-M.; et al. Mutations of the VHL tumour suppressor gene in renal carcinoma. Nat. Genet. 1994, 7, 85.

(26) Sumner, L. W.; Amberg, A.; Barrett, D.; Beale, M. H.; Beger, R.; Daykin, C. A.; Fan, T. W.-M.; Fiehn, O.; Goodacre, R.; Griffin, J. L.; Hankemeier, T.; Hardy, N.; Harnly, J.; Higashi, R.; Kopka, J.; Lane, A. N.; Lindon, J. C.; Marriott, P.; Nicholls, A. W.; Reily, M. D.; et al. Proposed minimum reporting standards for chemical analysis. Metabolomics 2007, 3, 211.

(27) Blaženović, I.; Kind, T.; Ji, J.; Fiehn, O. Software Tools and Approaches for Compound Identification of LC-MS/MS Data in Metabolomics. Metabolites 2018, 8, 31.

(28) Zheng, X.; Baker, H.; Hancock, W. S.; Fawaz, F.; McCaman, M.; Pungor, E., Jr. Proteomic analysis for the assessment of different lots of fetal bovine serum as a raw material for cell culture. Part IV. Application of proteomics to the manufacture of biological drugs. Biotechnol. Prog. 2006, 22, 1294.

(29) Martano, G.; Delmotte, N.; Kiefer, P.; Christen, P.; Kentner, D.; Bumann, D.; Vorholt, J. A. Fast sampling method for mammalian 
cell metabolic analyses using liquid chromatography-mass spectrometry. Nat. Protoc. 2015, 10, 1.

(30) Bateman, K. P.; Castro-Perez, J.; Wrona, M.; Shockcor, J. P.; Yu, K.; Oballa, R.; Nicoll-Griffith, D. A. MSE with mass defect filtering forin vitro andin vivo metabolite identification. Rapid Commun. Mass Spectrom. 2007, 21, 1485.

(31) McMillan, A.; Renaud, J. B.; Gloor, G. B.; Reid, G.; Sumarah, M. W. Post-acquisition filtering of salt cluster artefacts for LC-MS based human metabolomic studies. J. Cheminf. 2016, 8, 44.

(32) Thonusin, C.; IglayReger, H. B.; Soni, T.; Rothberg, A. E.; Burant, C. F.; Evans, C. R. Evaluation of intensity drift correction strategies using MetaboDrift, a normalization tool for multi-batch metabolomics data. J. Chromatogr. A 2017, 1523, 265.

(33) Kirwan, J. A.; Weber, R. J. M.; Broadhurst, D. I.; Viant, M. R. Direct infusion mass spectrometry metabolomics dataset: a benchmark for data processing and quality control. Sci. Data 2014, 1, 140012 .

(34) Trygg, J.; Wold, S. Orthogonal projections to latent structures (O-PLS). J. Chemom. 2002, 16, 119.

(35) Bylesjö, M.; Rantalainen, M.; Cloarec, O.; Nicholson, J. K.; Holmes, E.; Trygg, J. OPLS discriminant analysis: combining the strengths of PLS-DA and SIMCA classification. J. Chemom. 2006, 20, 341.

(36) Benjamini, Y.; Hochberg, Y. Controlling the False Discovery Rate: A Practical and Powerful Approach to Multiple Testing. J. R. Stat. Soc. Series B, Stat. Methodol. 1995, 57, 289.

(37) Wishart, D. S.; Tzur, D.; Knox, C.; Eisner, R.; Guo, A. C.; Young, N.; Cheng, D.; Jewell, K.; Arndt, D.; Sawhney, S.; Fung, C.; Nikolai, L.; Lewis, M.; Coutouly, M.-A.; Forsythe, I.; Tang, P.; Shrivastava, S.; Jeroncic, K.; Stothard, P.; Amegbey, G.; et al. HMDB: the Human Metabolome Database. Nucleic Acids Res. 2007, 35, D521. (38) Smith, C. A.; Maille, G. O.; Want, E. J.; Qin, C.; Trauger, S. A.; Brandon, T. R.; Custodio, D. E.; Abagyan, R.; Siuzdak, G. METLIN A metabolite mass spectral database. Ther. Drug Monit. 2005, 27, 747.

(39) Sud, M.; Fahy, E.; Cotter, D.; Brown, A.; Dennis, E. A.; Glass, C. K.; Merrill, A. H., Jr.; Murphy, R. C.; Raetz, C. R. H.; Russell, D. W.; Subramaniam, S. LMSD: LIPID MAPS structure database. Nucleic Acids Res. 2007, 35, D527.

(40) Creighton, C. J.; Morgan, M.; Gunaratne, P. H.; Wheeler, D. A.; Gibbs, R. A.; Gordon Robertson, A.; Chu, A.; Beroukhim, R.; Cibulskis, K.; Signoretti, S.; Vandin Hsin-Ta Wu, F.; Raphael, B. J.; Verhaak, R. G. W.; Tamboli, P.; Torres-Garcia, W.; Akbani, R.; Weinstein, J. N.; Reuter, V.; Hsieh, J. J.; Rose Brannon, A.; et al. Comprehensive molecular characterization of clear cell renal cell carcinoma. Nature 2013, 499, 43.

(41) Ricketts, C. J.; De Cubas, A. A.; Fan, H.; Smith, C. C.; Lang, M.; Reznik, E.; Bowlby, R.; Gibb, E. A.; Akbani, R.; Beroukhim, R.; Bottaro, D. P.; Choueiri, T. K.; Gibbs, R. A.; Godwin, A. K.; Haake, S.; Hakimi, A. A.; Henske, E. P.; Hsieh, J. J.; Ho, T. H.; Kanchi, R. S.; et al. The Cancer Genome Atlas Comprehensive Molecular Characterization of Renal Cell Carcinoma. Cell Rep. 2018, 23, 313.

(42) Daskalaki, E.; Pillon, N. J.; Krook, A.; Wheelock, C. E.; Checa, A. The influence of culture media upon observed cell secretome metabolite profiles: The balance between cell viability and data interpretability. Anal. Chim. Acta 2018, 1037, 338.

(43) Beger, R. D.; Dunn, W. B.; Bandukwala, A.; Bethan, B.; Broadhurst, D.; Clish, C. B.; Dasari, S.; Derr, L.; Evans, A.; Fischer, S.; Flynn, T.; Hartung, T.; Herrington, D.; Higashi, R.; Hsu, P.-C.; Jones, C.; Kachman, M.; Karuso, H.; Kruppa, G.; Lippa, K.; et al. Towards quality assurance and quality control in untargeted metabolomics studies. Metabolomics 2019, 15, 4.

(44) Domingo-Almenara, X.; Montenegro-Burke, J. R.; Benton, H. P.; Siuzdak, G. Annotation: A Computational Solution for Streamlining Metabolomics Analysis. Anal. Chem. 2018, 90, 480.

(45) Morgan, B.; Ezerina, D.; Amoako, T. N. E.; Riemer, J.; Seedorf, M.; Dick, T. P. Multiple glutathione disulfide removal pathways mediate cytosolic redox homeostasis. Nat. Chem. Biol. 2013, 9, 119.

(46) Ludwig, M.; Broeckling, C. D.; Dorrestein, P.; Dührkop, K.; Schymanski, E.; Boecker, S.; Nothias, L.-F. Mining the NIST Mass
Spectral Library Reveals the Extent of Sodium Assisted Inductive Cleavage in Collision-Induced Fragmentation. 2020, ChemRxiv:12114987.v1. Preprint.

(47) Miess, H.; Dankworth, B.; Gouw, A. M.; Rosenfeldt, M.; Schmitz, W.; Jiang, M.; Saunders, B.; Howell, M.; Downward, J.; Felsher, D. W.; Peck, B.; Schulze, A. The glutathione redox system is essential to prevent ferroptosis caused by impaired lipid metabolism in clear cell renal cell carcinoma. Oncogene 2018, 37, 5435.

(48) Ganti, S.; Taylor, S. L.; Kim, K.; Hoppel, C. L.; Guo, L.; Yang, J.; Evans, C.; Weiss, R. H. Urinary acylcarnitines are altered in human kidney cancer. Int. J. Cancer 2012, 130, 2791.

(49) Wettersten, H. I.; Hakimi, A. A.; Morin, D.; Bianchi, C.; Johnstone, M. E.; Donohoe, D. R.; Trott, J. F.; Aboud, O. A.; Stirdivant, S.; Neri, B.; Wolfert, R.; Stewart, B.; Perego, R.; Hsieh, J. J.; Weiss, R. H. Grade-Dependent Metabolic Reprogramming in Kidney Cancer Revealed by Combined Proteomics and Metabolomics Analysis. Cancer Res. 2015, 75, 2541.

(50) Mustafa, A.; Gupta, S.; Hudes, G. R.; Egleston, B. L.; Uzzo, R. G.; Kruger, W. D. Serum amino acid levels as a biomarker for renal cell carcinoma. J. Urol. 2011, 186, 1206.

(51) Zheng, H.; Ji, J.; Zhao, L.; Chen, M.; Shi, A.; Pan, L.; Huang, Y.; Zhang, H.; Dong, B.; Gao, H. Prediction and diagnosis of renal cell carcinoma using nuclear magnetic resonance-based serum metabolomics and self-organizing maps. Oncotarget 2016, 7, 59189.

(52) Hakimi, A. A.; Reznik, E.; Lee, C.-H.; Creighton, C. J.; Brannon, A. R.; Luna, A.; Aksoy, B. A.; Liu, E. M.; Shen, R.; Lee, W.; Chen, Y.; Stirdivant, S. M.; Russo, P.; Chen, Y.-B.; Tickoo, S. K.; Reuter, V. E.; Cheng, E. H.; Sander, C.; Hsieh, J. J. An Integrated Metabolic Atlas of Clear Cell Renal Cell Carcinoma. Cancer Cell 2016, 29, 104.

(53) Saito, K.; Arai, E.; Maekawa, K.; Ishikawa, M.; Fujimoto, H.; Taguchi, R.; Matsumoto, K.; Kanai, Y.; Saito, Y. Lipidomic Signatures and Associated Transcriptomic Profiles of Clear Cell Renal Cell Carcinoma. Sci. Rep. 2016, 6, 28932.

(54) Purohit, V.; Simeone, D. M.; Lyssiotis, C. A. Metabolic Regulation of Redox Balance in Cancer. Cancers 2019, 11, 955.

(55) Xiao, Y.; Meierhofer, D. Glutathione Metabolism in Renal Cell Carcinoma Progression and Implications for Therapies. Int. J. Mol. Sci. 2019, 20, 3672.

(56) Gorrini, C.; Harris, I. S.; Mak, T. W. Modulation of oxidative stress as an anticancer strategy. Nat. Rev. Drug Discov. 2013, 12, 931.

(57) Gao, J.; Aksoy, B. A.; Dogrusoz, U.; Dresdner, G.; Gross, B.; Sumer, S. O.; Sun, Y.; Jacobsen, A.; Sinha, R.; Larsson, E.; Cerami, E.; Sander, C.; Schultz, N. Integrative analysis of complex cancer genomics and clinical profiles using the cBioPortal. Sci. Signal. 2013, 6, pl1.

(58) Perillo, B.; Di Donato, M.; Pezone, A.; Di Zazzo, E.; Giovannelli, P.; Galasso, G.; Castoria, G.; Migliaccio, A. ROS in cancer therapy: the bright side of the moon. Exp. Mol. Med. 2020, 52, 192.

(59) Combs, J. A.; DeNicola, G. M. The Non-Essential Amino Acid Cysteine Becomes Essential for Tumor Proliferation and Survival. Cancers 2019, 11, 678.

(60) Rodrigues, D.; Monteiro, M.; Jerónimo, C.; Henrique, R.; Belo, L.; Bastos, M. d. L.; Guedes de Pinho, P.; Carvalho, M. Renal cell carcinoma: a critical analysis of metabolomic biomarkers emerging from current model systems. Transl. Res. 2017, 180, 1.

(61) Shibata, Y.; Yasui, H.; Higashikawa, K.; Miyamoto, N.; Kuge, Y. Erastin, a ferroptosis-inducing agent, sensitized cancer cells to X-ray irradiation via glutathione starvation in vitro and in vivo. PLoS One 2019, 14, No. e0225931.

(62) Cui, Q.; Wang, J.-Q.; Assaraf, Y. G.; Ren, L.; Gupta, P.; Wei, L.; Ashby, C. R.; Yang, D.-H.; Chen, Z.-S. Modulating ROS to overcome multidrug resistance in cancer. Drug Resist. Updates 2018, 41, 1.

(63) Gana, C. C.; Hanssen, K. M.; Yu, D. M. T.; Flemming, C. L.; Wheatley, M. S.; Conseil, G.; Cole, S. P. C.; Norris, M. D.; Haber, M.; Fletcher, J. I. MRP1 modulators synergize with buthionine sulfoximine to exploit collateral sensitivity and selectively kill MRP1-expressing cancer cells. Biochem. Pharmacol. 2019, 168, 237. 
(64) Briston, T.; Stephen, J. M.; Thomas, L. W.; Esposito, C.; Chung, Y.-L.; Syafruddin, S. E.; Turmaine, M.; Maddalena, L. A.; Greef, B.; Szabadkai, G.; Maxwell, P. H.; Vanharanta, S.; Ashcroft, M. VHL-Mediated Regulation of CHCHD4 and Mitochondrial Function. Front. Oncol. 2018, 8, 388.

(65) Cuperlovic-Culf, M.; Cormier, K.; Touaibia, M.; Reyjal, J.; Robichaud, S.; Belbraouet, M.; Turcotte, S. 1H NMR metabolomics analysis of renal cell carcinoma cells: Effect of VHL inactivation on metabolism. Int. J. Cancer 2016, 138, 2439.

(66) Wettersten, H. I.; Aboud, O. A.; Lara, P. N.; Weiss, R. H. Metabolic reprogramming in clear cell renal cell carcinoma. Nat. Rev. Nephrol. 2017, 13, 410.

(67) Zhao, Z.; Liu, Y.; Liu, Q.; Wu, F.; Liu, X.; Qu, H.; Yuan, Y.; Ge, J.; Xu, Y.; Wang, H. The mRNA Expression Signature and Prognostic Analysis of Multiple Fatty Acid Metabolic Enzymes in Clear Cell Renal Cell Carcinoma. J. Cancer 2019, 10, 6599.

(68) van der Mijn, J. C.; Fu, L.; Khani, F.; Zhang, T.; Molina, A. M.; Barbieri, C. E.; Chen, Q.; Gross, S. S.; Gudas, L. J.; Nanus, D. M. Combined Metabolomics and Genome-Wide Transcriptomics Analyses Show Multiple HIF $1 \alpha$-Induced Changes in Lipid Metabolism in Early Stage Clear Cell Renal Cell Carcinoma. Transl. Oncol. 2020, 13, 177.

(69) McGarry, J. D.; Mannaerts, G. P.; Foster, D. W. A possible role for malonyl-CoA in the regulation of hepatic fatty acid oxidation and ketogenesis. J. Clin. Invest. 1977, 60, 265.

(70) Carracedo, A.; Cantley, L. C.; Pandolfi, P. P. Cancer metabolism: fatty acid oxidation in the limelight. Nat. Rev. Cancer 2013, 13, 227.

(71) McGarry, J. D.; Brown, N. F. The Mitochondrial Carnitine Palmitoyltransferase System - From Concept to Molecular Analysis. Eur. J. Biochem. 1997, 244, 1.

(72) Neely, B. A.; Wilkins, C. E.; Marlow, L. A.; Malyarenko, D.; Kim, Y.; Ignatchenko, A.; Sasinowska, H.; Sasinowski, M.; Nyalwidhe, J. O.; Kislinger, T.; Copland, J. A.; Drake, R. R. Proteotranscriptomic Analysis Reveals Stage Specific Changes in the Molecular Landscape of Clear-Cell Renal Cell Carcinoma. PLoS One 2016, 11, No. e0154074.

(73) Sanchez, D. J.; Simon, M. C. Genetic and metabolic hallmarks of clear cell renal cell carcinoma. Biochim. Biophys. Acta, Rev. Cancer 2018, 1870, 23.

(74) Kim, Y.-S.; Jung, J.; Jeong, H.; Lee, J.-H.; Oh, H. E.; Lee, E. S.; Choi, J.-W. High Membranous Expression of Fatty Acid Transport Protein 4 Is Associated with Tumorigenesis and Tumor Progression in Clear Cell Renal Cell Carcinoma. Dis. Markers 2019, 2019, 5702026.

(75) Aiderus, A.; Black, M. A.; Dunbier, A. K. Fatty acid oxidation is associated with proliferation and prognosis in breast and other cancers. BMC Cancer 2018, 18, 805. 